\title{
Antibacterial Activity Exerted by some Diazabicyclo-steroid Derivatives Against Staphylococcus Aureus and Streptococcus Pneumoniae
}

\author{
HAU-HEREDIA LENIN ${ }^{1}$, FIGUEROA-VALVERDE LAURO ${ }^{1 *}$, GARCÍA-CERVERA ELODIA ${ }^{1}$, \\ LÓPEZ-RAMOS MARIA ${ }^{1 *}$, DÍAZ-CEDILLO FRANCISCO², POOL-GÓMEZ EDUARDO1, \\ ROSAS-NEXTICAPA MARCELA ${ }^{3}$, HERRERA-MEZA SOCORRO ${ }^{4}$ and \\ CAUICH-CARRILLO REGINA ${ }^{1}$
}

\begin{abstract}
${ }^{1}$ Laboratory of Pharmaco-Chemistry, Faculty of Chemical Biological Sciences, University, University Autonomous of Campeche, Av. Agustín Melgar s/n, Col Buenavista C.P. 24039 Campeche, Camp., México. ${ }^{2}$ Escuela Nacional de Ciencias Biológicas del Instituto Politécnico Nacional. Prol. Carpio y Plan de

Ayala s/n Col. Santo Tomas, México, D.F. C.P. 11340.

${ }^{3}$ Facultad de Nutrición, Universidad Veracruzana, Médicos y Odontologos s/n C.P. 91010, Unidad del Bosque Xalapa Veracruz, México.

${ }^{4}$ Instituto de Investigaciones Psicológicas, Universidad Veracruzana. Av. Dr. Luis Castelazo Ayala s/n Col Industrial Animas. C.P. 91190, Xalapa, Veracruz, México.

${ }^{\star}$ Corresponding author E-mail: Lauro_1999@yahoo
\end{abstract}

http://dx.doi.org/10.13005/ojc/330564

(Received: September 06, 2017; Accepted: October 04, 2017)

\begin{abstract}
We have synthesize three diazabicyclo-steroid derivatives to evaluate its antibacterial activity. This process involved a series of reactions such as; i) cycloaddition [2 + 2] of 5-hexyn-1ol to OTBS-testosterone (1) or progesterone (2) or OTBS-pregnenolone (3) to form cyclobuteneol-steroid derivatives ( 4 or 5 or 6 ); ii) the compounds 4 or 5 or 6 were reacted with ethylenediamine to form steroid-amino conjugates ( 7 or 8 or 9 ); iii) alkynylation of 7 or 8 or 9 with 5-hexyn-1-ol to form the steroid-amino-hexynol conjugates (10 or 11 or 12); iv) preparation of the cyclobuta-ynal-steroid derivatives (13 or 14 or 15 ) by the reaction of 10 or 11 or 12 with DMSO; v) amination of 13 or 14 or 15 with ethylenediamine to form new amino-steroid derivatives 16 or 19 or $21 ; \mathrm{vi})$ removal of the tert-butyldimethylsilyl from 16 or 21 with hydrofluoric acid to form hydroxyl-steroids (17 and 22); vii) preparation of 1,4-diazacycloundeca-5,11-dien-steroid derivatives by the reaction 17 or 19 or 22 with Copper(II) chloride. In order to evaluate the possibility of that compounds synthesized may have biological activity; in this study its antibacterial effect on Streptococcus pneumoniae and Staphylococcus aureus bacteria was evaluated. The results indicate that compound 20 exert higher antibacterial activity against Staphylococcus aureus and Streptococcus pneumoniae compared with 18 and 23 via interaction DNa-gyrase. In conclusion, these data indicate that antibacterial activity exerted by the compounds 20 depend of their structure chemical in comparison with the other steroid derivatives involved in this study.
\end{abstract}

Keywords:Testosterone, pregnenolone, progesterone steroid, derivatives, antibacterial. 


\section{INTRODUCTION}

Infectious diseases are one of the main causes of morbidity-mortality in the world ${ }^{1,2}$. There are several reports which indicate that some causal agents, such as Staphylococcus aureus ${ }^{3}$, Streptococcus pneumoniae ${ }^{4}$ and other sare involved in infection diseases developed. Although there are many therapeutic agents for treatment of these bacterial microorganisms ${ }^{5,6}$, unfortunately prolonged antibiotic therapy induces bacterial-resistance, because some bacteria have developed ways to circumvent the effects of antibiotics ${ }^{7,8}$. Therefore, antibiotic resistance to bacteria can be considered a serious threat for the human health; this fact requires an international approach to its management. In this sense, new drugs have been developed for control of bacterial resistance; for example, the preparation of diazabicycle derivatives as antibacterial agents against Staphylococcus aureus strains ${ }^{9}$ Other data in dicate the synthesis and antibacterial activity of some 1,3-diazabicyclo-carbapen derivatives 2,4-dichloro-5-fluorophenyl derivatives against Staphylococcus aureus strain ${ }^{10}$. In addition, some 3, 7-diazabicyclo[3.3.1]nonaneazines were prepared as antibacterial agents against Staphylococcus aureus ${ }^{11}$. In addition a study showed the synthesis of 9-alkyl-I,5-diazabicyclo-[4.3.0]non-5-enes which decreased the bacterial growth of Staphylococcus aureus ${ }^{12}$. Also, other report showed the preparation of 1,4-diazabicyclo-[2.2.2]octane with antibacterial activity against a Staphylococcus aureus strain ${ }^{13}$. Additionaly, other report shown that the diazabycycle derivative (1,3-diazaadamante) exerted antibacterial activity against Staphylococcus aureus $^{14}$.

On the other hand, a 3,7-diazabicyclo[3.3.1]nonane derivative was synthesized as antibacterial agent against Streptococcus pneumoniae ${ }^{15,16}$. Additionally, other data showed the synthesis of the compound 3,8Diazahicyclo[3.2.I] octane ${ }^{17}$ which inhibits the bacterial growth of Streptococcus pneumoniae ${ }^{18,19}$. All these experimental results show that several diazabicyclo derivatives can induce antibacterial effects against Staphylococcus aureusand Streptococcus pneumoniae. Analyzing these data, in this study three diazabicyclo-steroid derivatives were synthesized and their antibacterial activity against Staphylococcus aureusandStreptococcus pneumoniae was evaluated in vitro.

\section{EXPERIMENTAL}

\section{General methods}

The testosterone derivative and pregnenolone (OTBS-Testosterone and OTBDSpregnenolone) were prepared using methods previously reported ${ }^{20}$. The other reagents used in this study were purchased from Sigma-Aldrich Co. Ltd. The melting point was determined on an Electrothermal (900 model). ${ }^{1} \mathrm{H}$ and ${ }^{13} \mathrm{C}$ NMR spectra were recorded on a Varian VXR-300/5 FT NMR spectrometer at 300 and $75.4 \mathrm{MHz}$ in $\mathrm{CDCl}_{3}$ using TMS as internal standard. EIMS spectra were obtained with a Finnigan Trace GCPolaris Q. spectrometer. Elementary analysis data were acquired from a Perkin Elmer Ser. II CHNS/0 2400 elemental analyzer.

\section{Preparation of cyclobuta-3-one-steroid derivatives}

A solution of 1 or 2 or $3(0.50 \mathrm{mmol})$, 5-hexyn-1-ol (60 $\mu \mathrm{l}, 0.54 \mathrm{mmol})$, Iron(III) chloride anhydrous ( $80 \mathrm{mg}, 0.49 \mathrm{mmol}$ ) in $5 \mathrm{ml}$ of methanol was stirring for $72 \mathrm{~h}$ to room temperature. The reaction mixture was evaporated to dryness under reduced pressure. After, the residue was purified by crystallization from methanol: water(4:1).

8-(tert-Butyl-dimethyl-silanyloxy)-2-(4-Hydroxy-butyl)5a,7a-dimethyl-4,5,5a,5b,6,7,7a,8,9,10,10a,10b,11,12tetradecahydro-2aH-cyclobuta[j]cyclopenta [a]phenanthren-3-one (4)

yielding $67 \%$ of product, m.p. $74-76^{\circ} \mathrm{C}$; IR $\left(\mathrm{V}_{\max }, \mathrm{cm}^{-1}\right): 3400,1720$ and 1058; ${ }^{1} \mathrm{H}$ NMR (300 $\left.\mathrm{MHz}, \mathrm{CDCl}_{3}\right) \delta_{\mathrm{H}}: 0.08(\mathrm{~s}, 6 \mathrm{H}), 0.68(\mathrm{~s}, 3 \mathrm{H}), 0.86(\mathrm{~s}$, $9 \mathrm{H}), 0.94-1.04(\mathrm{~m}, 2 \mathrm{H}), 1.08(\mathrm{~s}, 3 \mathrm{H}), 1.20-1.48(6 \mathrm{H})$, $152-1.60(\mathrm{~m}, 4 \mathrm{H}), 1.62-2.10(\mathrm{~m}, 8 \mathrm{H}), 2.20(\mathrm{~m}, 2 \mathrm{H})$, 2.22-3.54 (m, 5H), 3.60 (broad, $1 \mathrm{H}), 3.66(\mathrm{~m}, 2 \mathrm{H})$, $5.70(\mathrm{~d}, 1 \mathrm{H}, \mathrm{J}=1.82 \mathrm{~Hz}) \mathrm{ppm} .{ }^{13} \mathrm{C}$ NMR $(75.4 \mathrm{~Hz}$, $\left.\mathrm{CDCl}_{3}\right) \delta_{\mathrm{C}}:-4.60(\mathrm{C}-24, \mathrm{C}-31), 11.32(\mathrm{C}-21), 14.80$ (C-20), 18.02 (C-32), 21.34 (C-18), 23.52 (C-8), 24.30 (C-27), 25.24 (C-11), 25.50 (C-33, C-34, C-35), 31.06 (C-7), 31.82 (C-10), 32.44 (C-12), 32.54 (C-28), 36.54 (C-3), 36.70 (C-19), 37.02 (C26), 38.50 (C-13), 40.40 (C-9), 42.24 (C-1), 43.30 (C-5), 49.78 (C-2), 52.00 (C-4), 60.16 (C-15), 62.55 (C-29), 81.62 (C-6), 134.52 (C-17), 147.68 (C-16), 
210.62 (C-14) ppm. El-MS m/z: 500.36 Anal. Calcd. for $\mathrm{C}_{31} \mathrm{H}_{52} \mathrm{O}_{3} \mathrm{Si}: \mathrm{C}, 74.34 ; \mathrm{H}, 10.47 ; \mathrm{O}, 9.58 ;$ Si, 5.61 . Found: C, 74.26; $\mathrm{H}, 10.36$.

8-Acetyl-2-(4-hydroxy-butyl)-5a,7a-dimethyl$4,5,5 a, 5 b, 6,7,7 a, 8,9,10,10 a, 10 b, 11,12$ tetradecahydro-2aH-cyclobuta[j]cyclopenta[a] phenanthren-3-one (5)

yielding $44 \%$ of product, m.p. $80-82^{\circ} \mathrm{C}$; IR $\left(\mathrm{V}_{\text {max }}, \mathrm{cm}^{-1}\right): 3404$ and 1722; ${ }^{1} \mathrm{H}$ NMR (300 MHz, $\left.\mathrm{CDCl}_{3}\right) \delta_{\mathrm{H}}: 0.70(\mathrm{~s}, 3 \mathrm{H}), 1.06(\mathrm{~s}, 3 \mathrm{H}), 1.20-1.46(8 \mathrm{H})$, 1.50-1.58 (m, 4H), 1.66-2.10 (m, 8H), $2.12(\mathrm{~s}, 3 \mathrm{H})$, $2.20(\mathrm{t}, 2 \mathrm{H}, \mathrm{J}=13.20 \mathrm{~Hz}), 2.22-3.00(\mathrm{~m}, 5 \mathrm{H}), 3.60$ (broad, 1H), 3.66 (t, 2H, J = $11.00 \mathrm{~Hz}), 5.70(\mathrm{~d}, 1 \mathrm{H}$, $\mathrm{J}=1.32 \mathrm{~Hz})$ ppm. ${ }^{13} \mathrm{C}$ NMR $\left(75.4 \mathrm{~Hz}, \mathrm{CDCl}_{3}\right) \delta_{\mathrm{C}}$ : 13.40 (C-21), 14.80 (C-20), 21.52 (C-7), 23.02 (C-18), 24.30 (C-24), 24.44 (C-17), 25.24 (C-10), 30.74 (C-30), 31.82 (C-9), 32.44 (C-11), 32.54 (C25), 35.64 (C-3), 37.02 (C-23), 38.48 (C-12), 39.02 (C-6), 40.40 (C-8), 42.24 (C-1), 44.28 (C-5), 51.19 (C-2), 57.72 (C-4), 60.16 (C-14), 62.55 (C-26), 63.98 (C-19), 134.52 (C-16), 147.68 (C-15), 208.28 (C-28), 210.62 (C-13) ppm. El-MS m/z: 412.29 Anal. Calcd. for $\mathrm{C}_{27} \mathrm{H}_{40} \mathrm{O}_{3}$ : C, 78.60; $\mathrm{H}, 9.77 ; \mathrm{O}, 11.63$. Found: C, 78.52; H, 9.68.

1-[8-(tert-Butyl-dimethyl-silanyloxy)-10-(4-hydroxybutyl)-3a, 3 b-dimethyl-1, 2, 3, 3a, 4, 5, 5a, $5 \mathrm{~b}, 6,7,11 \mathrm{a}, 12,12 \mathrm{a}, 12 \mathrm{~b}-\mathrm{hexadecahydro-}$ cyclobuta[k]cyclopenta[a]phenanthren-3yl]ethanonone (6)

yielding $56 \%$ of product, m.p. 148-150 ${ }^{\circ} \mathrm{C}$; IR $\left(\mathrm{V}_{\max }, \mathrm{cm}^{-1}\right): 3402,1720$ and 1058; ${ }^{1} \mathrm{H}$ NMR $\left(300 \mathrm{MHz} \mathrm{CDCl}_{3}\right) \delta_{\mathrm{H}}: 0.06(\mathrm{~s}, 6 \mathrm{H}), 0.70(\mathrm{~s}, 3 \mathrm{H}), 0.90$ (s, 9H), $0.98(\mathrm{~s}, 3 \mathrm{H}), 1.14-1.46(\mathrm{~m}, 9 \mathrm{H}), 1.50(\mathrm{~m}, 2 \mathrm{H})$, $1.52(\mathrm{~m}, 1 \mathrm{H}), 1.56-2.00(\mathrm{~m}, 7 \mathrm{H}), 2.02(\mathrm{~m}, 2 \mathrm{H}), 2.12(\mathrm{~s}$, $3 \mathrm{H}), 2.16-2.20(\mathrm{~m}, 3 \mathrm{H}), 2.58-3.50(\mathrm{~m} \mathrm{5H}), 3.60$ (broad, 1H), 3.66 (t, 2H, J = $11.00 \mathrm{~Hz}), 5.40$ (d, 1H,J = 1.32 Hz) ppm. ${ }^{13} \mathrm{C}$ NMR $\left(75.4 \mathrm{~Hz}, \mathrm{CDCl}_{3}\right) \delta_{\mathrm{C}}:-4.60$ (C-22, C-32),13.70 (C-24), 16.90 (C-24), 18.50 (C-33),21.22 (C-10),24.12(C-15),24.20 (C-16), 24.90(C-28), 26.10 (C-34,C-36, C-37), 28.34 (C35), 28.52 (C2) ,32.54(C-29),32.88 (C-6), 33.34 (C-14), 35.00 (C-3), 35.14 (C-11), 35.62 (C-27) ,38 .68 (C4) ,39.02(C-9),43.20 (C-13), 44.14 (C-8), 44.80 (C-5), 51.12 (C-12), 56.42 (C-7), 62.55 (C-30), 63.78 (C-17), 71.56 (C-1), 133.92 (C-19), 153.90 (C-18), 208.58 (C-25) ppm. El-MS m/z: 528.39 Anal. Calcd. for $\mathrm{C}_{33} \mathrm{H}_{56} \mathrm{O}_{3} \mathrm{Si}: \mathrm{C}, 74.94 ; \mathrm{H}, 10.67 ; \mathrm{O}, 9.08 ; \mathrm{Si}, 5.31$. Found: C, 74.83; $\mathrm{H}, 10.54$.
Preparation of cyclobutene-1-ol-steroid-amino conjugates

A solution of 4 or 5 or $6(0.50 \mathrm{mmol})$, ethylenediamine $(60 \mu \mathrm{l}, 0.90 \mathrm{mmol})$ and boric acid (50 mg, $0.80 \mathrm{mmol}$ ), in $5 \mathrm{ml}$ of methanol was stirring for $72 \mathrm{~h}$ to room temperature. The reaction mixture was evaporated to dryness under reduced pressure. After, the residue was purified by crystallization from methanol: water(3:1).

4[3-(2-Amino-ethylimino)-8-(tert-butyl-dimethylsilanyloxy)-5a,7a-dimethyl-2a,3,4,5,5a,5b, $6,7,7 a, 8,9,10,10 a, 10 b, 11,12$-hexadecahydrocyclobuta[j]cyclopenta[a]phenan- thren-2yl]butan-1-ol (7)

yielding $55 \%$ of product, m.p. $120-122{ }^{\circ} \mathrm{C}$; IR $\left(\mathrm{V}_{\max }, \mathrm{cm}^{-1}\right)$ : 3402, 3370 and 1058; ${ }^{1} \mathrm{H}$ NMR (300 $\left.\mathrm{MHz} \mathrm{CDCl}_{3}\right) \delta_{\mathrm{H}}: 0.08(\mathrm{~s}, 6 \mathrm{H}), 0.68(\mathrm{~s}, 3 \mathrm{H}), 0.88(\mathrm{~s}$, $9 \mathrm{H}), 0.94(\mathrm{~m}, 1 \mathrm{H}), 0.98(\mathrm{~s}, 3 \mathrm{H}), 1.06-1.48(8 \mathrm{H}), 1.50$ $(\mathrm{m}, 2 \mathrm{H}), 1.56-1.60(\mathrm{~m}, 2 \mathrm{H}), 1.62(\mathrm{~m}, 2 \mathrm{H}), 1.64-1.88$ $(\mathrm{m}, 5 \mathrm{H}), 2.15(\mathrm{t}, 2 \mathrm{H}, \mathrm{J}=13.20 \mathrm{~Hz}), 2.22-2.42(\mathrm{~m}$, $3 \mathrm{H}), 3.10-3.50(\mathrm{~m}, 4 \mathrm{H}), 3.54(\mathrm{~m}, 1 \mathrm{H}), 3.66(\mathrm{t}, 2 \mathrm{H}$, $\mathrm{J}=11.00 \mathrm{~Hz}$ ), 4.10 (broad, 3H), $5.20(\mathrm{~m}, 1 \mathrm{H}), 5.40$ (d, $1 \mathrm{H}, \mathrm{J}=1.32 \mathrm{~Hz})$ ppm. ${ }^{13} \mathrm{C}$ NMR $\left(75.4 \mathrm{~Hz}, \mathrm{CDCl}_{3}\right)$ $\delta_{\mathrm{C}}$ : -4.60 (C-24, C-34), 11.34 (C-21), 15.60 (C-20), 18.02 (C-35), 21.32 (C-18), 23.52 (C-8), 24.38 (C-30), 25.14 (C-11), 25.50 (C-36, C-37, C-38), 26.64 (C-12), 27.22 (C-13), 31.04 (C-7), 32.02 (C-10), 32.54 (C-31), 36.50 (C-29), 36.54 (C-3), 36.70 (C-19), 41.00 (C-27), 42.04 (C-9), 43.30 (C-5), 45.16 (C-1), 47.40 (C-2), 49.08 (C-15), 52.00 (C-4), 53.60 (C-26), 62.55 (C-32), 81.70 (C-6), 132.34 (C-17), 150.38 (C-16), 169.34 (C-14) ppm. El-MS m/z: 542.42 Anal. Calcd. for $\mathrm{C}_{33} \mathrm{H}_{58} \mathrm{~N}_{2} \mathrm{O}_{2} \mathrm{Si}$ : C, 73.01; H, 10.77; N, 5.16; O, 5.89; Si, 5.17. Found: C, 73.00; H, 10.68.

4-\{3-(2-Amino-ethylimino)-8-[1-(2-aminoethylimino)-ethyl]-5a,7a-dimethyl-2a,3,4,5,5a,5b, $6,7,7 a, 8,9,10,10 a, 10 b, 11,12-h e x a d e c a h y d r o-$ cyclobuta[j]cyclopenta[a]phenan- thren-2-yl\}butan-1-ol (8)

yielding $64 \%$ of product, m.p. $70-72{ }^{\circ} \mathrm{C}$; IR $\left(\mathrm{V}_{\max }, \mathrm{cm}^{-1}\right)$ : 3402 and 3332; ${ }^{1} \mathrm{H}$ NMR (300 MHz, $\left.\mathrm{CDCl}_{3}\right) \delta_{\mathrm{H}}: 0.88(\mathrm{~s}, 3 \mathrm{H}), 0.98(\mathrm{~s}, 3 \mathrm{H}), 1.20-1.44(\mathrm{~m}$, $7 \mathrm{H}), 1.50(\mathrm{~m}, 2 \mathrm{H}), 1.52-1.58(\mathrm{~m}, 3 \mathrm{H}), 1.62(\mathrm{~m}, 2 \mathrm{H})$, $1.76(\mathrm{~m}, 1 \mathrm{H}), 1.80(\mathrm{~s}, 3 \mathrm{H}), 1.82-2.12(\mathrm{~m}, 4 \mathrm{H}), 2.18$ (t, $2 \mathrm{H}, \mathrm{J}=13.20 \mathrm{~Hz}), 2.22-2.40(\mathrm{~m}, 5 \mathrm{H}), 3.09(\mathrm{~m}$, $2 \mathrm{H}), 3.10-3.50(\mathrm{~m}, 4 \mathrm{H}), 3.52(\mathrm{~m}, 2 \mathrm{H}), 3.66(\mathrm{t}, 2 \mathrm{H}, \mathrm{J}$ $=11.00 \mathrm{~Hz}), 4.20($ broad, $5 \mathrm{H}), 5.20(\mathrm{~m}, 1 \mathrm{H}), 5.40(\mathrm{~d}$, 
$1 \mathrm{H}, \mathrm{J}=1.82 \mathrm{~Hz})$ ppm. ${ }^{13} \mathrm{C} \mathrm{NMR}\left(75.4 \mathrm{~Hz}, \mathrm{CDCl}_{3}\right) \delta_{\mathrm{C}}$ : 13.20 (C-21), 15.60 (C-20), 16.70 (C-36), 21.92 (C-7), 24.40 (C-27), 25.18 (C-10), 25.28 (C-17), 26.40 (C-18), 26.62 (C-11), 27.28 (C-12), 32.04 (C-9), 32.54 (C-28), 36.52 (C-26), 36.56 (C-3), 38.28 (C-6), 41.00 (C-24), 41.02 (C-34), 42.04 (C-8), 42.82 (C-5), 45.17 (C-1), 48.80 (C-2), 49.06 (C-14), 53.10 (C-33), 53.60 (C-23), 56.22 (C-4), 62.55 (C-29), 63.08 (C-19), 132.32 (C-16), 150.38 (C-15), 156.78 (C-31), 169.28 (C-13) ppm. El-MS m/z 496.41 Anal. Calcd. for $\mathrm{C}_{31} \mathrm{H}_{52} \mathrm{~N}_{4} \mathrm{O}: \mathrm{C}, 74.95 ; \mathrm{H}, 10.55 ; \mathrm{N}, 11.28$; $\mathrm{O}, 3.22$. Found: $\mathrm{C}, 74.84 ; \mathrm{H}, 10.46$.

4-[3-[1-(2-Amino-ethylimino)-ethyl]-8-(isopropyldimethyl-silan yloxy)-3a, 5 b-dimeth yl$1,2,3,3 a, 4,5,5 a, 5 b, 6,7,8,9,11 a, 12,12 a, 12 b-$ hexadecah ydro-cyclobuta[k]cyclopenta [a]phenanthren- 10-yl]-butan-1-ol (9)

yielding $45 \%$ of product, m.p. $258-260^{\circ} \mathrm{C}$; IR $\left(\mathrm{V}_{\text {max }}, \mathrm{cm}^{-1}\right):$ 3370, 3330 and 1060; ${ }^{1} \mathrm{H}$ NMR (300 $\left.\mathrm{MHz}, \mathrm{CDCl}_{3}\right) \quad \delta_{\mathrm{H}}: 0.07(\mathrm{~s}, 6 \mathrm{H}), 0.88(\mathrm{~s}, 3 \mathrm{H}), 0.90$ $(\mathrm{s}, 9 \mathrm{H}), 0.98(\mathrm{~s}, 3 \mathrm{H}), 1.14-1.40(\mathrm{~m}, 7 \mathrm{H}), 1.50(\mathrm{~m}$, $2 \mathrm{H}), 1.51(\mathrm{~m}, 1 \mathrm{H}), 1.52(\mathrm{~m}, 2 \mathrm{H}), 1.53-1.78(\mathrm{~m}, 6 \mathrm{H})$, $1.80(\mathrm{~s}, 3 \mathrm{H}), 1.82-1.94(\mathrm{~m}, 2 \mathrm{H}), 2.00(\mathrm{~m}, 2 \mathrm{H}), 2.12-$ $2.40(\mathrm{~m}, 5 \mathrm{H}), 3.08-3.52(\mathrm{~m}, 4 \mathrm{H}), 3.53(\mathrm{~m}, 1 \mathrm{H}), 3.66$ (t, 2H, J = $11.00 \mathrm{~Hz}$ ), 4.10 (broad, 3H), 5.40 (d, 1H, $\mathrm{J}=1.82 \mathrm{~Hz})$ ppm. ${ }^{13} \mathrm{C} \mathrm{NMR}\left(75.4 \mathrm{~Hz}, \mathrm{CDCl}_{3}\right) \delta_{\mathrm{C}}:-$ 4.60 (C-22, C-35), 13.20 (C-24), 16.70 (C-38), 16.90 (C-23), 18.44 (C-36), 21.68 (C-10), 24.90 (C-31), 25.22 (C-15), 26.04 (C-37, C-39, C-40), 26.40 (C-16), 28.52 (C-2), 32.54 (C-32), 33.30 (C-14), 33.80 (C-6), 35.00 (C-3), 35.14 (C-11), 35.57 (C-30), 38.28 (C-9), 38.60 (C-4), 41.00 (C-28), 42.82 (C-8), 43.15 (C-13), 44.80 (C-5), 51.14 (C-12), 53.10 (C-27), 57.36 (C-7), 62.55 (C-33), 63.08 (C-17), 71.56 (C-1), 133.94 (C-19), 153.88 (C-18), 156.70 (C-25) ppm. El-MS m/z570.45 Anal. Calcd. for $\mathrm{C}_{35} \mathrm{H}_{62}$ $\mathrm{N}_{2} \mathrm{O}_{2} \mathrm{Si}: \mathrm{C}, 73.63 ; \mathrm{H}, 10.95 ; \mathrm{N}, 4.91 ; \mathrm{O}, 5.60 ;$ Si, 4.92. Found: C, 73.52; H, 10.83.

\section{Alkynylation of amino groups}

A solution of 7 or 8 or $9(0.50 \mathrm{mmol})$, 5-hexyn-1-ol (130 $\mu \mathrm{l}, 1.18 \mathrm{mmol})$ and cupric chloride (120 mg, $0.89 \mathrm{mmol}$ ), in $5 \mathrm{ml}$ of methanol was stirring for $72 \mathrm{~h}$ to room temperature. The reaction mixture was evaporated to dryness under reduced pressure. After, the residue was purified by crystallization from hexane:methanol: water $(1: 4: 2)$.
6-\{2-[8-tert-Butyl-dimethyl-silanyloxy)-2-(3hydroxy-propyl)-5a,7a-dimethyl-4,5,5a,5b,6,7,7a, $8,9,10,10 a, 10 b, 11,12$-tetradecahydro-2a $H$ cyclobuta[j] cyclopenta [a]phenanthren-3ylideneamino]-ethylamino\}-hex-5-yn-1-ol (10) yielding $38 \%$ of product, m.p. $130-132{ }^{\circ} \mathrm{C}$; IR $\left(\mathrm{V}_{\max }, \mathrm{cm}^{-1}\right)$ : 3400 and 1058; ${ }^{1} \mathrm{H}$ NMR $(300 \mathrm{MHz}$, $\left.\mathrm{CDCl}_{3}\right) \delta_{\mathrm{H}}: 0.06(\mathrm{~s}, 6 \mathrm{H}), 0.68(\mathrm{~s}, 3 \mathrm{H}), 0.88(\mathrm{~s}, 9 \mathrm{H})$, $0.90(\mathrm{~m}, 1 \mathrm{H}), 0.98(\mathrm{~s}, 3 \mathrm{H}), 1.04-1.56(9 \mathrm{H}), 1.58(\mathrm{~m}$, $2 \mathrm{H}), 1.60-1.62(\mathrm{~m}, 2 \mathrm{H}), 1.64(\mathrm{~m}, 2 \mathrm{H}), 1.68(\mathrm{~m}, 2 \mathrm{H})$, 1.78-2.22 (m, 6H), $2.26(\mathrm{~m}, 2 \mathrm{H}), 2.30(\mathrm{~m}, 2 \mathrm{H}), 2.40$ $(\mathrm{m}, 1 \mathrm{H}), 3.20(\mathrm{t}, 2 \mathrm{H}, \mathrm{J}=13.60 \mathrm{~Hz}), 3.52(\mathrm{~m}, 2 \mathrm{H})$, $3.53(\mathrm{~m}, 1 \mathrm{H}), 3.56(\mathrm{~m}, 2 \mathrm{H}), 3.66(\mathrm{t}, 2 \mathrm{H}, \mathrm{J}=11.00$ $\mathrm{Hz}$ ), 3.80 (broad, $3 \mathrm{H}), 5.40$ (d, $1 \mathrm{H}, \mathrm{J}=1.32 \mathrm{~Hz}), 5.60$ $(\mathrm{m}, 1 \mathrm{H}) \mathrm{ppm} .{ }^{13} \mathrm{C} \mathrm{NMR}\left(75.4 \mathrm{~Hz}, \mathrm{CDCl}_{3}\right) \delta_{\mathrm{C}}:-4.60$ (C-22, C-40), 11.40 (C-23), 15.60 (C-24), 16.18 (C-31), 17.80 (C-41), 21.32 (C-13), 23.14 (C-37), 23.54 (C-11), 25.14 (C-9), 25.72 (C-42, C-43, C44), 25.80 (C-32), 26.64 (C-14), 27.22 (C-15), 30.06 (C-33), 31.04 (C-10), 32.02 (C-8), 33.40 (C-36), 36.50 (C-4), 36.72 (C-12), 42.04 (C-7), 43.30 (C-2), 45.16 (C-6), 47.40 (C-5), 47.68 (C-17), 51.72 (C-26), 51.98 (C-3), 54.14 (C-27), 61.80 (C-38), 62.05 (C-34), 78.44 (C-30), 81.66 (C-1), 87.30 (C29), 133.52 (C-18), 150.58 (C-16), 169.34 (C-16) ppm. El-MS m/z: 624.46 Anal. Calcd. for $\mathrm{C}_{38} \mathrm{H}_{64} \mathrm{~N}_{2} \mathrm{O}$ Si: C, 73.02; $\mathrm{H}, 10.32 ; \mathrm{N}, 4.48 ; \mathrm{O}, 7.68$; Si, 4.49. Found: C, 73.00; H, 10.26.

6- $\{2-[8-\{1-[2-(6-H y d r o x y-h e x-1-y n y l a m i n o)-$ ethylimino]-ethyl\}-2-(3-hydroxy-propyl)-5a,7adimethyl-4,5,5a,5b,6,7,7a,8,9,10,10a,10b,11,12tetradecahydro-2aH-cyclobuta[j] cyclopenta[a] phenanthren-3-ylideneamino]-ethylamino\}-hex5-yn-1-ol (11)

yielding $64 \%$ of product, m.p. $133-134{ }^{\circ} \mathrm{C}$; $\mathrm{IR}\left(\mathrm{V}_{\max }, \mathrm{cm}^{-1}\right)$ : 3402 and 3330; ${ }^{1} \mathrm{H}$ NMR $(300 \mathrm{MHz}$, $\left.\mathrm{CDCl}_{3}\right) \delta_{\mathrm{H}}: 0.88(\mathrm{~s}, 3 \mathrm{H}), 0.98(\mathrm{~s}, 3 \mathrm{H}), 1.20-1.56(\mathrm{~m}$, $10 \mathrm{H}), 1.58(\mathrm{~m}, 4 \mathrm{H}), 1.66(\mathrm{~m}, 4 \mathrm{H}), 1.68(\mathrm{~s}, 3 \mathrm{H}), 1.69$ $(\mathrm{m}, 2 \mathrm{H}), 1.78-2.22(\mathrm{~m}, 7 \mathrm{H}), 2.26(\mathrm{t}, 2 \mathrm{H}, \mathrm{J}=13.20$ $\mathrm{Hz}), 2.28(\mathrm{~m}, 1 \mathrm{H}), 2.30(\mathrm{~m}, 4 \mathrm{H}), 2.38-2.40(\mathrm{~m}, 2 \mathrm{H})$ $3.16(\mathrm{~m}, 4 \mathrm{H}), 3.52(\mathrm{t}, 2 \mathrm{H}, \mathrm{J}=11.00 \mathrm{~Hz}), 3.56(\mathrm{~m}$, $4 \mathrm{H}), 3.64(\mathrm{~m}, 4 \mathrm{H}), 3.70$ (broad, $5 \mathrm{H}), 5.40(\mathrm{~d}, 1 \mathrm{H}, \mathrm{J}=$ $1.82 \mathrm{~Hz}), 5.60(\mathrm{~m}, 1 \mathrm{H}) \mathrm{ppm} .{ }^{13} \mathrm{C}$ NMR $(75.4 \mathrm{~Hz}$, $\left.\mathrm{CDCl}_{3}\right) \delta_{\mathrm{C}}: 13.20$ (C-20), 15.60 (C-21), 16.20 (C-29, C-40), 21.92 (C-10), 22.60 (C-49), 23.20 (C-46), 25.18 (C-8), 25.80 (C-30, C-41), 26.40 (C-12), 26.62 (C-13), 26.64 (C-14), 27.28 (C-15), 30.02 (C-31, C-42), 32.04 (C-7), 33.42 (C-45), 36.52 (C-3), 38.18 (C-9), 42.06 (C-8), 42.82 (C-1), 45.17 (C-5), 47.68 
(C-17), 48.80 (C-4), 51.22 (C-24), 51.76 (C-35), 52.44 (C-2), 54.10 (C-25, C-36), 56.70 (C-11), 61.80 (C-47), 62.05 (C-32, C-43), 78.48 (C-28, C-39), 87.28 (C-27, C-38), 133.52 (C-19), 150.58 (C-18), 162.68 (C-22), 169.28 (C-16) ppm. El-MS $\mathrm{m} / \mathrm{z} 674.51$ Anal. Calcd. for $\mathrm{C}_{42} \mathrm{H}_{66} \mathrm{~N}_{4} \mathrm{O}_{3}: \mathrm{C}, 74.73$; $\mathrm{H}, 9.86$; N, 8.30; O, 7.11. Found: C, 74.64; H, 9.78.

6-(2-\{1-[8-(tert-Butyl-dimethyl-silanyloxy)-10-(4hydroxy-butyl)-3a,5b-dimethyl-1,2,3, 3a,4,5,5a, 5b, 6,7,8,9, 11a,12,12a,12b-hexadecahydrocyclobuta [k]cyclopenta[a] phenanthren-3-yl]ethylideneamino\}-ethylamino)-hex-5-yn-1-ol (12) yielding $64 \%$ of product, m.p. $82-84{ }^{\circ} \mathrm{C}$; IR $\left(\mathrm{V}_{\max }, \mathrm{cm}^{-1}\right):$ 3400, 3332 and 1058; ${ }^{1} \mathrm{H}$ NMR (300 $\left.\mathrm{MHz}, \mathrm{CDCl}_{3}\right) \delta_{\mathrm{H}}: 0.06(\mathrm{~s}, 6 \mathrm{H}), 0.88(\mathrm{~s}, 3 \mathrm{H}), 0.90$ $(\mathrm{s}, 9 \mathrm{H}), 0.98(\mathrm{~s}, 3 \mathrm{H}), 1.16-1.42(\mathrm{~m}, 7 \mathrm{H}), 1.50$ $(\mathrm{m}, 2 \mathrm{H}), 1.51(\mathrm{~m}, 1 \mathrm{H}), 1.52(\mathrm{~m}, 2 \mathrm{H}), 1.53(\mathrm{~m}, 1 \mathrm{H})$, $1.58(\mathrm{~m}, 2 \mathrm{H}), 1.59(\mathrm{~m}, 1 \mathrm{H}), 1.64(\mathrm{~m}, 2 \mathrm{H}), 1.59(\mathrm{~m}$, $1 \mathrm{H}), 1.64(\mathrm{~m}, 2 \mathrm{H}), 1.66(\mathrm{~m}, 1 \mathrm{H}), 1.68(\mathrm{~s}, 3 \mathrm{H}), 1.70-$ $1.96(\mathrm{~m}, 5 \mathrm{H}), 2.00(\mathrm{~m}, 2 \mathrm{H}), 2.14-2.28(\mathrm{~m}, 4 \mathrm{H}), 2.30$ $(\mathrm{m}, 2 \mathrm{H}), 2.40(\mathrm{~m}, 1 \mathrm{H}), 3.18(\mathrm{~m}, 2 \mathrm{H}), 3.50(\mathrm{~m}, 1 \mathrm{H})$, $3.54(\mathrm{~m}, 2 \mathrm{H}), 3.58$ (broad, $3 \mathrm{H}), 3.66(\mathrm{~m}, 2 \mathrm{H}), 3.67$ $(\mathrm{m}, 2 \mathrm{H}), 5.40(\mathrm{~d}, 1 \mathrm{H}, \mathrm{J}=1.32 \mathrm{~Hz}) \mathrm{ppm} .{ }^{13} \mathrm{C} \mathrm{NMR}$ $\left(75.4 \mathrm{~Hz}, \mathrm{CDCl}_{3}\right) \delta_{\mathrm{C}}:-4.56(\mathrm{C}-22, \mathrm{C}-42), 13.20$ (C-24), 16.20 (C-32), 16.90 (C-23), 18.44 (C-43), 21.68 (C-10), 22.62 (C-45), 24.90 (C-38), 25.82 (C33), 26.10 (C-44, C-46, C-47), 26.40 (C-16), 26.60 (C-15), 28.50 (C-2), 30.10 (C-34), 32.54 (C-39), 33.30 (C-14), 33.80 (C-6), 35.00 (C-3), 35.14 (C11), 35.57 (C-37), 38.18 (C-9), 38.64 (C-4), 42.82 (C-8), 43.15 (C-13), 44.80 (C-5), 51.12 (C-12), 51.24 (C-27), 53.60 (C-7), 54.16 (C-28), 56.70 (C-17), 62.08 (C-35), 62.50 (C-40), 71.52 (C-1), 78.44 (C31), 87.32 (C-30), 133.84 (C-19), 153.88 (C-18), 162.70 (C-25) ppm. El-MS m/z 666.51 Anal. Calcd. for $\mathrm{C}_{41} \mathrm{H}_{70} \mathrm{~N}_{2} \mathrm{O}_{3} \mathrm{Si}: \mathrm{C}, 73.82 ; \mathrm{H}, 10.58 ; \mathrm{N}, 4.20 ; \mathrm{O}, 7.20$; $\mathrm{Si}, 4.21$. Found: $\mathrm{C}, 73.74 ; \mathrm{H}, 10.44$.

Preparation of cyclobuta-ynal-steroid derivatives A solution of 10 or 11 or $12(0.50$ $\mathrm{mmol}$ ), in $5 \mathrm{ml}$ of dimethyl sulfoxyde was stirring for $72 \mathrm{~h}$ to room temperature. The reaction mixture was evaporated to dryness under reduced pressure. After, $5 \mathrm{ml}$ of water was added and stirring for $72 \mathrm{~h}$ to room temperature. The residue was purified by crystallization from hexane:methanol: water (1:4:2).
7-\{2-[8-(tert-Butyl-dimethyl-silanyloxy)-5a,7adimethyl-2-(4-oxo-butyl)-4,5,5a,5b,6,7,7a, $8,9,10,10 a, 10 b, 11,12$-tetradecahydro-2aHcyclobuta[j] cyclopenta[a] phenanthren-3ylideneamino]-ethylamino\}-hept-6-ynal (13)

yielding $44 \%$ of product, m.p. $50-52^{\circ} \mathrm{C}$; IR $\left(\mathrm{V}_{\max }, \mathrm{cm}^{-1}\right): 3332$ and 1058; ${ }^{1} \mathrm{H}$ NMR (300 MHz, $\left.\mathrm{CDCl}_{3}\right) \delta_{\mathrm{H}}: 0.08(\mathrm{~s}, 6 \mathrm{H}), 0.69(\mathrm{~s}, 3 \mathrm{H}), 0.84(\mathrm{~s}, 9 \mathrm{H})$, $0.92(\mathrm{~m}, 1 \mathrm{H}), 0.98(\mathrm{~s}, 3 \mathrm{H}), 1.02-1.62(11 \mathrm{H}), 1.70$ $(\mathrm{m}, 2 \mathrm{H}), 1.78-1.82(\mathrm{~m}, 3 \mathrm{H}), 1.84(\mathrm{~m}, 2 \mathrm{H}), 1.86(\mathrm{~m}$, $1 \mathrm{H}), 1.94(\mathrm{~m}, 2 \mathrm{H}), 2.20-2.22(\mathrm{~m}, 2 \mathrm{H}), 2.24(\mathrm{~m}, 2 \mathrm{H})$, 2.26-2.30 (m, 4H), $2.40(\mathrm{~m}, 1 \mathrm{H}), 2.56(\mathrm{~m}, 2 \mathrm{H}), 3.20$ (t, $2 \mathrm{H}, \mathrm{J}=13.34 \mathrm{~Hz}), 3.52(\mathrm{~m}, 1 \mathrm{H}), 3.56(\mathrm{t}, 2 \mathrm{H}, \mathrm{J}=$ $8.79 \mathrm{~Hz}), 5.20$ (broad, 1H), $5.22(\mathrm{~m}, 1 \mathrm{H}), 5.40$ (d, $1 \mathrm{H}, \mathrm{J}=1.32 \mathrm{~Hz}), 9.70(\mathrm{CHO}), 9.82\left(\mathrm{CO}_{2} \mathrm{H}\right) \mathrm{ppm} .{ }^{13} \mathrm{C}$ NMR $\left(75.4 \mathrm{~Hz}, \mathrm{CDCl}_{3}\right) \delta_{\mathrm{C}}:-4.60$ (C-22, C-42), 11.40 (C-23), 15.60 (C-24), 16.18 (C-31), 17.80 (C-43), 21.00 (C-38), 21.30 (C-13), 22.14 (C-33), 23.54 (C11), 25.14 (C-9), 25.70 (C-44, C-45, C-46), 26.64 (C-14), 26.94 (C-32), 27.22 (C-15), 31.02 (C-10), 32.06 (C-8), 35.50 (C-37), 36.56 (C-4), 36.70 (C12), 42.04 (C-7), 43.30 (C-2), 43.52 (C-39), 44.60 (C-34), 45.16 (C-6), 47.40 (C-5), 49.08 (C-17), 51.72 (C-26), 51.98 (C-3), 54.14 (C-27), 79.64 (C-30), 81.66 (C-1), 87.30 (C-29), 132.38 (C-19), 147.80 (C-18), 169.34 (C-16), 202.18 (C-40), 202.44 (C35)ppm.El-MS $\mathrm{m} / \mathrm{z}$ : 648.46 Anal.Calcd.for $\mathrm{C}_{40} \mathrm{H}_{64} \mathrm{~N}_{2} \mathrm{O}_{3} \mathrm{Si}: \mathrm{C}, 74.02 ; \mathrm{H}, 9.94 ; \mathrm{N}, 4.32 ; \mathrm{O}, 7.40$, Si, 4.33. Found: C, 74.00; $\mathrm{H}, 9.88$.

7-[2-(5a,7a-Dimethyl-2-(4-oxo-butyl)-8-\{1-[2-(7-oxohept-1 -ynylamino)-ethylimino] ethyl\} $4,5,5 a, 5 b, 6,7,7 a, 8,9,10,10 a, 10 b, 11,12$ tetradecahydro-2aH-cyclobuta[j]cyclopenta[a] phenanthren-3-ylideneamino]-ethylamino]-hept-6ynal (14)

yielding $64 \%$ of product, m.p. $48-50{ }^{\circ} \mathrm{C}$; IR $\left(\mathrm{V}_{\max }, \mathrm{cm}^{-1}\right)$ : 3330 and 1742; ${ }^{1} \mathrm{H}$ NMR $(300 \mathrm{MHz}$, $\left.\mathrm{CDCl}_{3}\right) \delta_{\mathrm{H}}: 0.88(\mathrm{~s}, 3 \mathrm{H}), 0.98(\mathrm{~s}, 3 \mathrm{H}), 1.20-1.56(\mathrm{~m}$, $10 \mathrm{H}), 1.68(\mathrm{~s}, 3 \mathrm{H}), 1.70(\mathrm{~m}, 4 \mathrm{H}), 1.78-1.84(\mathrm{~m}, 7 \mathrm{H})$, $1.86(\mathrm{~m}, 4 \mathrm{H}), 1.94(\mathrm{~m}, 1 \mathrm{H}), 1.96(\mathrm{~m}, 2 \mathrm{H}), 2.12-2.22$ $(\mathrm{m}, 3 \mathrm{H}), 2.24(\mathrm{~m}, 4 \mathrm{H}), 2.26(\mathrm{~m}, 2 \mathrm{H}), 2.28(\mathrm{~m}, 1 \mathrm{H})$, $2.30(\mathrm{~m}, 2 \mathrm{H}), 2.38-2.40(\mathrm{~m}, 2 \mathrm{H}), 2.56(\mathrm{~m}, 4 \mathrm{H}), 3.16$ $(\mathrm{m}, 4 \mathrm{H}), 3.56(\mathrm{~m}, 4 \mathrm{H}), 5.20$ (broad, $5 \mathrm{H}), 5.22(\mathrm{~m}$, $1 \mathrm{H}), 5.40(\mathrm{~d}, 1 \mathrm{H}, \mathrm{J}=1.82 \mathrm{~Hz}), 9.70(\mathrm{CHO}), 9.82\left(\mathrm{CO}_{2} \mathrm{H}\right)$ ppm. ${ }^{13} \mathrm{C} \mathrm{NMR}\left(75.4 \mathrm{~Hz}, \mathrm{CDCl}_{3}\right) \delta_{\mathrm{C}}: 13.20$ (C-20), 15.60 (C-21), 16.12 (C-29, C-41), 21.00 (C-48), 21.92 (C10), 22.10 (C-31, C-43), 22.60 (C-52), 25.18 (C-8), 26.40 (C- 
12), 26.62 (C-13), 26.64 (C-14), 26.90 (C-30, C-42), 27.28 (C-15), 32.04 (C-7), 35.54 (C-47), 36.52 (C-3), 38.18 (C9), 42.06 (C-6), 42.82 (C-1), 43.50 (C-49), 44.62 (C-32, C-44), 45.17 (C-5), 48.80 (C-4), 49.02 (C-17), 51.22 (C-24), 51.76 (C-36), 52.44 (C-2), 54.10 (C-25, C-37), 56.70 (C-11), 79.60 (C-28, C-40), 87.28 (C-27, C-39), 132.40 (C-19), 147.80 (C-18), 162.68 (C-22), 169.28 (C-16), 202.20 (C-50), 202.42 (C-33, C-45), ppm. El-MS m/z710.51 Anal. Calcd. for $\mathrm{C}_{45} \mathrm{H}_{66} \mathrm{~N}_{4} \mathrm{O}_{3}: \mathrm{C}, 76.01 ; \mathrm{H}, 9.36 ; \mathrm{N}$, 7.88; O, 6.75. Found: C, 76.00; $\mathrm{H}, 9.28$.

6-(2-\{1-[8-(tert-Butyl-dimethyl-silanyloxy)-3a,5bdimethyl-10-(5-oxo-pentyl)-1,2,3,3a,4, $5,5 a, 5 b, 6,7,8,9,11 a, 12,12 a, 12 b-h e x a d e c a h y d r o-$ cyclobuta[k]cyclopenta[a]phenan-thren-3yl]ethylideneamino\}-ethylamino)hex-5-ynal (15) yielding $64 \%$ of product, m.p. $148-150{ }^{\circ} \mathrm{C}$; IR $\left(\mathrm{V}_{\max }, \mathrm{cm}^{-1}\right)$ : 3332, 1749 and 1182; ${ }^{1} \mathrm{H}$ NMR $(300 \mathrm{MHz}$, $\left.\mathrm{CDCl}_{3}\right) \delta_{\mathrm{H}}: 0.06(\mathrm{~s}, 6 \mathrm{H}), 0.88(\mathrm{~s}, 3 \mathrm{H}), 0.90(\mathrm{~s}, 9 \mathrm{H}), 0.98(\mathrm{~s}$, $3 \mathrm{H}), 1.14-1.40(\mathrm{~m}, 7 \mathrm{H}), 1.46(\mathrm{~m}, 2 \mathrm{H}), 1.50-1.52(\mathrm{~m}, 2 \mathrm{H})$, $1.56(\mathrm{~m}, 2 \mathrm{H}), 1.58-1.66(\mathrm{~m}, 2 \mathrm{H}), 1.68(\mathrm{~s}, 3 \mathrm{H}), 1.70-1.82$ $(\mathrm{m}, 4 \mathrm{H}), 1.84(\mathrm{~m}, 2 \mathrm{H}), 1.94(\mathrm{~m}, 1 \mathrm{H}), 1.96(\mathrm{~m}, 2 \mathrm{H}), 2.12-$ $2.28(\mathrm{~m}, 4 \mathrm{H}), 2.34(\mathrm{~m}, 2 \mathrm{H}), 2.40(\mathrm{~m}, 1 \mathrm{H}), 2.44-2.45(\mathrm{~m}$, $4 \mathrm{H}), 3.18(\mathrm{~m}, 2 \mathrm{H}), 3.50(\mathrm{~m}, 1 \mathrm{H}), 3.56(\mathrm{~m}, 2 \mathrm{H}), 5.20$ (broad, $1 \mathrm{H}), 5.40$ (d, $1 \mathrm{H}, \mathrm{J}=1.32 \mathrm{~Hz}), 9.66(\mathrm{~d}, 1 \mathrm{H}, \mathrm{J}=1.90 \mathrm{~Hz})$, $9.72(\mathrm{~d}, 1 \mathrm{H}, \mathrm{J}=1.90 \mathrm{~Hz}) \mathrm{ppm} .{ }^{13} \mathrm{CNMR}\left(75.4 \mathrm{~Hz}, \mathrm{CDCl}_{3}\right) \delta_{\mathrm{C}}$ : -4.60 (C-22, C-43), 13.20 (C-24), 15.20(C-32), 16.90 (C23), 18.44 (C-44), 21.68 (C-10), 21.72 (C-33) , 22.62 (C46), 23.00 (C-39), 25.30 (C-38), 26.06 (C-45, C-47, C48), 26.40 (C-16), 26.60 (C-15) , 28.52 (C-2), 33.30 (C14), 33.80 (C-6), 35.00 (C-3), 35.17 (C-11), 35.52 (C37), 38.18 (C-9), 38.66(C-4), 42.80(C-8), 43.12 (C-34), 43.17 (C-13), 43.69 (C-40), 44.80 (C-5), 51.14 (C12), 51.26 (C-27), 53.60 (C-7), 54.16 (C-28), 56.70 $(\mathrm{C}-17), 71.56(\mathrm{C}-1), 75.90(\mathrm{C}-31), 87.32$ (C30),133.94 (C-19), 154.08 (C-18), 162.70 (C-25), 198.62 (C-35), 202.48 (C-41) ppm. El-MS m/z 676.49 Anal. Calcd. for $\mathrm{C}_{42} \mathrm{H}_{68} \mathrm{~N}_{2} \mathrm{O}_{3} \mathrm{Si}: \mathrm{C}, 74.50 ; \mathrm{H}$, 10.12; N, 4.14; O, 7.09; Si, 4.15. Found: C, 74.42; H, 10.02 .

Amination of cyclobuta-ynal-steroid derivatives A solution of 13 or 14 or $15(0.50 \mathrm{mmol})$, ethylene diamine $(60 \mu \mathrm{l}, 0.90 \mathrm{mmol})$ and boric acid (50 $\mathrm{mg}, 0.80 \mathrm{mmol}$ ), in $5 \mathrm{ml}$ of methanol was stirring for $72 \mathrm{~h}$ to room temperature. The reaction mixture was evaporated to dryness under reduced pressure. After, the residue was purified by crystallization from methanol:water (3:1).
2-[4-(2-Amino-ethylimino)-butyl]-3-\{2-[7-(2-aminoethylimino)-hept-1-ynylamino]-ethylimino\}-8-(tertbutyl-dimethyl-silanyloxy)-5a,7a-dimethyl$2 a, 3,4,5,5 a, 5 b, 6,7,7 a, 8,9,10,10 a, 10 b, 11,12-$ hexadecahydro-cyclobuta[j]cyclopenta[a] phenanthrene (16).

yielding $63 \%$ of product, m.p. $102-104{ }^{\circ} \mathrm{C}$; IR $\left(\mathrm{V}_{\max }, \mathrm{cm}^{-1}\right): 3378,3330$ and 1058; ${ }^{1} \mathrm{H}$ NMR (300 $\left.\mathrm{MHz} \mathrm{CDCl}_{3}\right) \delta_{\mathrm{H}}: 0.08(\mathrm{~s}, 6 \mathrm{H}), 0.69(\mathrm{~s}, 3 \mathrm{H}), 0.84$ $(\mathrm{s}, 9 \mathrm{H}), 0.92(\mathrm{~m}, 1 \mathrm{H}), 0.98(\mathrm{~s}, 3 \mathrm{H}), 1.04-1.56(9 \mathrm{H})$, $1.58(\mathrm{~m}, 2 \mathrm{H}), 1.60-1.62(\mathrm{~m}, 2 \mathrm{H}), 1.64(\mathrm{~m}, 2 \mathrm{H}), 1.74$ $(\mathrm{m}, 2 \mathrm{H}), 1.78-1.88(\mathrm{~m}, 4 \mathrm{H}), 2.04(\mathrm{~m}, 2 \mathrm{H}), 2.20(\mathrm{~m}$, $4 \mathrm{H}), 2.21-2.24(\mathrm{~m}, 2 \mathrm{H}), 2.28(\mathrm{~m}, 2 \mathrm{H}), 2.36(\mathrm{~m}, 2 \mathrm{H})$, $2.40(\mathrm{~m}, 1 \mathrm{H}), 3.10(\mathrm{~m}, 4 \mathrm{H}), 3.20(\mathrm{t}, 2 \mathrm{H}, \mathrm{J}=13.34$ $\mathrm{Hz}), 3.52(\mathrm{~m}, 4 \mathrm{H}), 3.53(\mathrm{~m}, 1 \mathrm{H}) .3 .56(\mathrm{t}, 2 \mathrm{H}, \mathrm{J}=8.79$ $\mathrm{Hz}), 4.52$ (broad, 5H), $5.22(\mathrm{~m}, 1 \mathrm{H}), 5.40(\mathrm{~d}, 1 \mathrm{H}, \mathrm{J}=$ $1.32 \mathrm{~Hz}), 7.70(\mathrm{~m}, 1 \mathrm{H}), 8.20(\mathrm{~m}, 1 \mathrm{H}) \mathrm{ppm} .{ }^{13} \mathrm{C} \mathrm{NMR}$ $\left(75.4 \mathrm{~Hz}, \mathrm{CDCl}_{3}\right) \delta_{\mathrm{C}}:-4.60$ (C-24, C-48), 11.40 (C-21), 15.60 (C-20), 16.38 (C-31), 17.98 (C-49), 21.30 (C-18), 23.54 (C-8), 24.92 (C-34), 25.14 (C-11), 25.22 (C-41), 25.50 (C-50, C-51, C-52), 26.64 (C-12), 26.94 (C-33), 27.22 (C-13), 27.40 (C-32), 31.02 (C-7), 31.10 (C-42), 32.06 (C-10), 36.10 (C-40), 36.56 (C-3), 36.70 (C-19), 40.54 (C-38, C-46), 42.02 (C-9), 43.30 (C-5), 45.16 (C-1), 47.40 (C-2), 49.08 (C-15), 51.62 (C-37), 51.70 (C-26), 51.98 (C-4), 54.14 (C-27), 58.00 (C-45), 79.64 (C-30), 81.66 (C-6), 87.30 (C-29), 132.38 (C-17), 152.24 (C-43), 156.18 (C-35), 156.22 (C-16), 169.34 (C-14) ppm. El-MS m/z: 732.58 Anal. Calcd. for $\mathrm{C}_{44} \mathrm{H}_{76} \mathrm{~N}_{6} \mathrm{OSi}$ : C, 72.08; $\mathrm{H}, 10.45 ; \mathrm{N}, 11.46$; O, 2.18, Si, 3.83. Found: C, 72.00; H, 10.34.

(Z)-7-((2-Aminoethyl)imino)-N-(2-(((Z)-1 ((5aR,7aS,(S,E)-2-(E)-4-((2-aminoethyl)-imino)butyl)-3[2-(((Z)-7-((2-aminoethyl)imino)hept-1-yn-1yl)amino)ethyl)imino)-5a,7a-dimethyl$2 a, 3,4,5,5 a, 5 b, 6,7,7 a, 8,9,10,10 a, 10 b, 11,12-$ hexadecahydro-cyclobuta[j]cyclopenta[a] phenanthren-8-yl)ethylidene)amino)ethyl)hept-1-yn1-amine (19).

yielding $44 \%$ of product, m.p. $84-86{ }^{\circ} \mathrm{C}$; IR $\left(\mathrm{V}_{\max }, \mathrm{cm}^{-1}\right)$ : 3378 and 3330; ${ }^{1} \mathrm{H}$ NMR $\left(300 \mathrm{MHz} \mathrm{CDCl}_{3}\right)$ $\delta_{\mathrm{H}}: 0.88(\mathrm{~s}, 3 \mathrm{H}), 0.98(\mathrm{~s}, 3 \mathrm{H}), 1.20-1.56(\mathrm{~m}, 10 \mathrm{H}), 1.58(\mathrm{~m}$, $4 \mathrm{H}), 1.66(\mathrm{~m}, 4 \mathrm{H}), 1.68(\mathrm{~s}, 3 \mathrm{H}), 1.74(\mathrm{~m}, 2 \mathrm{H}), 1.78-1.94$ $(\mathrm{m}, 4 \mathrm{H}), 2.06(\mathrm{~m}, 2 \mathrm{H}), 2.12(\mathrm{~m}, 1 \mathrm{H}), 2.22(\mathrm{~m}, 4 \mathrm{H}), 2.21-$ $2.28(\mathrm{~m}, 3 \mathrm{H}), 2.30(\mathrm{~m}, 2 \mathrm{H}), 2.36(\mathrm{~m}, 4 \mathrm{H}), 2.39-2.40(\mathrm{~m}$, $2 \mathrm{H}), 3.10(\mathrm{~m}, 6 \mathrm{H}), 3.16(\mathrm{~m}, 4 \mathrm{H}), 3.52(\mathrm{~m}, 4 \mathrm{H}), 3.56(\mathrm{~m}$, 4H), 4.60 (broad, 8H), $5.22(\mathrm{~m}, 1 \mathrm{H}), 5.40(\mathrm{~d}, 1 \mathrm{H}, \mathrm{J}=1.82$ $\mathrm{Hz}), 7.70(\mathrm{~m}, 2 \mathrm{H}), 8.20(\mathrm{~m}, 1 \mathrm{H}) \mathrm{ppm} .{ }^{13} \mathrm{C}$ NMR $(75.4$ 
$\left.\mathrm{Hz}, \mathrm{CDCl}_{3}\right) \delta_{\mathrm{C}}: 13.20$ (C-21), 15.60 (C-20), 16.42 (C-28, C-52), 21.92 (C-7), 22.60 (C-61), 24.90 (C-31, C-55), 25.18 (C-10), 25.22 (C-17, C-38), 26.40 (C-18), 26.62 (C-11), 27.00 (C-30, C-54), 27.28 (C-12), 27.44 (C-29, C-53), 31.10 (C-39), 32.04 (C-9), 36.14 (C-37), 36.52 (C-3), 38.28 (C-6), 40.56 (C-35, C-43, C-59), 42.02 (C-8), 42.82 (C-5), 45.20 (C-1), 48.80 (C-2), 49.02 (C-14), 51.22 (C-47), 51.60 (C-34, C-58), 51.76 (C-23), 54.10 (C-24, C-48), 56.20 (C-4), 56.70 (C-19), 58.00 (C-42), 79.60 (C-27, C-51), 87.28 (C-26, C-50), 132.40 (C-16), 152.26 (C-40), 156.20 (C-32, C-56), 156.28 (C-15), 162.68 (C-45), 169.28 (C-13) ppm. El-MS $m / z$ 836.68 Anal.Calcd.for $\mathrm{C}_{51} \mathrm{H}_{84} \mathrm{~N}_{10}$ : C, 73.16; H, 10.11; N, 16.73. Found: C, 73.07; $\mathrm{H}, 10.04$.

(E)-6-((2-Aminoethyl)imino)- N-(2-(((Z)-1((3R,3aS,5bR,8S)-10-((E)-5-((2-aminoethyl)imino)pentyl)-8-(((tert-butyldimethylsilyl)oxy)3a,5b-dimethyl-1, 2, 3, 3a, 5,5a,5b ,6,7,8,9,11a,1, 12a,12 bhexadecahydrocyclobuta [k] cyclopenta [a]phenanthren-3-yl)ethylidene)amino)ethyl)hex1-yn-1-amine (21).

yielding $45 \%$ of product, m.p. $142-144{ }^{\circ} \mathrm{C}$; IR $\left(\mathrm{V}_{\max }, \mathrm{cm}^{-1}\right): 3378,3330$ and 1058; ${ }^{1} \mathrm{H}$ NMR (300 $\left.\mathrm{MHz}, \mathrm{CDCl}_{3}\right) \delta_{\mathrm{H}}: 0.06(\mathrm{~s}, 6 \mathrm{H}), 0.88(\mathrm{~s}, 3 \mathrm{H}), 0.90(\mathrm{~s}$, $9 \mathrm{H}), 0.98(\mathrm{~s}, 3 \mathrm{H}), 1.14-1.38(\mathrm{~m}, 6 \mathrm{H}), 1.40(\mathrm{~m}, 2 \mathrm{H})$, 1.41-1.66 (m, 5H), $1.68(\mathrm{~s}, 3 \mathrm{H}), 1.70(\mathrm{~m}, 1 \mathrm{H}), 1.76$ $(\mathrm{m}, 2 \mathrm{H}), 1.78-1.84(\mathrm{~m}, 3 \mathrm{H}), 1.90(\mathrm{~m}, 2 \mathrm{H}), 1.94$ $(\mathrm{m}, 1 \mathrm{H}), 2.06-2.10(\mathrm{~m}, 4 \mathrm{H}), 2.12(\mathrm{~m}, 2 \mathrm{H}), 2.14-2.40$ $(\mathrm{m}, 5 \mathrm{H}), 2.42(\mathrm{~m}, 2 \mathrm{H}), 3.10(\mathrm{~m}, 4 \mathrm{H}), 3.18(\mathrm{~m}, 2 \mathrm{H})$, $3.52(\mathrm{~m}, 4 \mathrm{H}), 3.54(\mathrm{~m}, 1 \mathrm{H}), 3.56(\mathrm{~m}, 2 \mathrm{H}), 4.52$ (broad, $5 \mathrm{H}), 5.40(\mathrm{~d}, 1 \mathrm{H}, \mathrm{J}=1.32 \mathrm{~Hz}), 8.10((\mathrm{~m}, 2 \mathrm{H}) \mathrm{ppm}$. ${ }^{13} \mathrm{C}$ NMR $\left(75.4 \mathrm{~Hz}, \mathrm{CDCl}_{3}\right) \delta_{\mathrm{C}}:-4.60(\mathrm{C}-24, \mathrm{C}-49)$, 13.20 (C-21), 16.80 (C-32), 16.90 (C-20), 18.44 (C-50), 21.68 (C-7), 22.62 (C-52), 25.30 (C-15), 26.06 (C-51, C-53, C-54), 26.40 (C-16), 26.80 (C-41), 27.46 (C-33), 27.82 (C-42), 28.50 (C-9), 30.30 (C-34), 30.40 (C-43), 33.30 (C-14), 33.80 (C-3), 35.00 (C-8), 35.17 (C-11), 35.82 (C-40), 38.28 (C-6), 38.66 (C-1), 40.50 (C-38, C-47), 42.80 (C-5), 43.17 (C-13), 44.80 (C-2), 51.14 (C-12), 51.26 (C-27), 54.16 (C-28), 56.70 (C-17), 57.34 (C-4), 58.00 (C-37, C-46), 71.56 (C-10), 86.46 (C-31), 87.32 (C-30), 133.94 (C-19), 154.08 (C-18), 154.50 (C-35, C-44), 162.70 (C-25) ppm. El-MS m/z 760.61 Anal. Calcd. for $\mathrm{C}_{46} \mathrm{H}_{80} \mathrm{~N}_{6} \mathrm{OSi}$ : C, 72.58; $\mathrm{H}, 10.59 ; \mathrm{N}$, 11.04; O, 2.10; Si, 3.69. Found: C, 72.42; H, 10.42.
Removal of the tert-butyldimethylsilylane fragment of 16 or 21.

A solution of 16 or $21(0.50 \mathrm{mmol})$ in $5 \mathrm{ml}$ of hydrofluoric acid was stirring for $72 \mathrm{~h}$ to room temperature. The reaction mixture was evaporated to dryness under reduced pressure. After, the residue was purified by crystallization from methanol: water (3:1).

2-[4-(2-Amino-ethylimino)butyl]-3-\{2-[7-(2amino-ethylimino)-hept-1-ynylamino]ethylimino\}-5a,7a-dimethyl-2a,3,4,5,5a,5b ,6,7,7a,8,9,10,10a,10b,11,12-hexadecahydrocyclobuta[j]cyclopenta[a]phenanthren-8-ol (17). yielding $55 \%$ of product, m.p. $100-102{ }^{\circ} \mathrm{C}$; IR $\left(\mathrm{V}_{\max }, \mathrm{cm}^{-1}\right): 3400,3380$ and 3330; ${ }^{1} \mathrm{H}$ NMR (300 $\left.\mathrm{MHz} \mathrm{CDCl}_{3}\right) \delta_{\mathrm{H}}: 0.84(\mathrm{~s}, 3 \mathrm{H}), 0.94(\mathrm{~m}, 1 \mathrm{H}), 0.98(\mathrm{~s}$, $3 \mathrm{H}), 1.10-1.56(10 \mathrm{H}), 1.58(\mathrm{~m}, 2 \mathrm{H}), 1.62(\mathrm{~m}, 1 \mathrm{H})$, $1.66(\mathrm{~m}, 2 \mathrm{H}), 1.74(\mathrm{~m}, 2 \mathrm{H}), 1.78-1.98(\mathrm{~m}, 4 \mathrm{H}), 2.04$ $(\mathrm{m}, 2 \mathrm{H}), 2.20(\mathrm{~m}, 2 \mathrm{H}), 2.21-2.24(\mathrm{~m}, 2 \mathrm{H}), 2.30(\mathrm{~m}$, $2 \mathrm{H}), 2.36(\mathrm{~m}, 2 \mathrm{H}), 2.40(\mathrm{~m}, 1 \mathrm{H}), 3.10(\mathrm{~m}, 4 \mathrm{H}), 3.20$ $(\mathrm{t}, 2 \mathrm{H}, \mathrm{J}=13.34 \mathrm{~Hz}), 3.52(\mathrm{~m}, 4 \mathrm{H}), 3.56(\mathrm{t}, 2 \mathrm{H}$, $\mathrm{J}=8.79 \mathrm{~Hz}), 3.64(\mathrm{~m}, 1 \mathrm{H}), 4.80$ (broad, $6 \mathrm{H}), 5.22$ $(\mathrm{m}, 1 \mathrm{H}), 5.40(\mathrm{~d}, 1 \mathrm{H}, \mathrm{J}=1.32 \mathrm{~Hz}), 7.70(\mathrm{~m}, 1 \mathrm{H})$, $8.20(\mathrm{~m}, 1 \mathrm{H}) \mathrm{ppm} .{ }^{13} \mathrm{C} \mathrm{NMR}\left(75.4 \mathrm{~Hz}, \mathrm{CDCl}_{3}\right) \delta_{\mathrm{C}}$ : 11.80 (C-21), 15.60 (C-20), 16.38 (C-29), 21.30 (C-18), 23.74 (C-8), 24.92 (C-32), 25.14 (C-11), 25.22 (C-39), 26.64 (C-12), 26.94 (C-31), 27.22 (C-13), 27.40 (C-30), 30.72 (C-7), 31.10 (C-40), 32.06 (C-10), 36.10 (C-38), 36.56 (C-3), 37.30 (C-19), 40.54 (C-36, C-44), 42.02 (C-9), 44.00 (C-5), 45.16 (C-1), 47.40 (C-2), 49.08 (C-15), 51.12 (C-4), 51.62 (C-35), 51.78 (C-24), 54.14 (C-25), 58.00 (C-43), 79.64 (C-28), 81.80 (C-6), 87.30 (C-27), 132.38 (C-17), 152.24 (C-41), 156.18 (C33), 156.22 (C-16), 169.34 (C-14) ppm. El-MS m/z: 618.49 Anal. Calcd. for $\mathrm{C}_{38} \mathrm{H}_{62} \mathrm{~N}_{6} \mathrm{O}: \mathrm{C}, 73.74 ; \mathrm{H}$, 10.10; N, 13.58; O, 2.58. Found: C, 73.66; H, 10.04 .

$3-(1-\{2-[6-(2-A m$ inoethylimino $)-$ hex - 1 ynylamino]ethylimino\}-ethyl)-10-[5-(2-aminoethylimino)-penthyl]-3a,5 b-dimethyl$1,2,3,3 a 4,5,5 a, 5 b, 6,7,8,9,11 a, 12,12 a, 12 b-$ hexadecahydrocyclobuta[k]cyclopenta[a] phenanthren-8-ol (22).

yielding $38 \%$ of product, m.p. $122-124^{\circ} \mathrm{C}$; IR $\left(\mathrm{V}_{\text {max }}, \mathrm{cm}^{-1}\right)$ : 3402 and 33323; ${ }^{1} \mathrm{H}$ NMR (300 MHz, $\left.\mathrm{CDCl}_{3}\right) \delta_{\mathrm{H}}: 0.88(\mathrm{~s}, 3 \mathrm{H}), 0.98(\mathrm{~s}, 3 \mathrm{H}), 1.14-1.38(\mathrm{~m}$, $6 \mathrm{H}), 1.40(\mathrm{~m}, 2 \mathrm{H}), 1.42-1.66(\mathrm{~m}, 5 \mathrm{H}), 1.68(\mathrm{~s}, 3 \mathrm{H})$, 
1.70-1.74 (m, 2H), $1.76(\mathrm{~m}, 2 \mathrm{H}), 1.78-1.84(\mathrm{~m}, 2 \mathrm{H})$, $1.90(\mathrm{~m}, 2 \mathrm{H}), 1.94(\mathrm{~m}, 1 \mathrm{H}), 2.06-2.08(\mathrm{~m}, 4 \mathrm{H}), 2.12$ $(\mathrm{m}, 2 \mathrm{H}), 2.14-2.40(\mathrm{~m}, 5 \mathrm{H}), 2.42(\mathrm{~m}, 2 \mathrm{H}), 3.10(\mathrm{~m}$, $4 \mathrm{H}), 3.18(\mathrm{~m}, 2 \mathrm{H}), 3.52(\mathrm{~m}, 4 \mathrm{H}), 3.56(\mathrm{~m}, 2 \mathrm{H}), 3.80$ $(\mathrm{m}, 1 \mathrm{H}), 4.24$ (broad, $6 \mathrm{H}), 6.00(\mathrm{~d}, 1 \mathrm{H}, \mathrm{J}=1.00 \mathrm{~Hz})$, $7.70(\mathrm{~m}, 1 \mathrm{H}), 8.10(\mathrm{~m}, 1 \mathrm{H}) \mathrm{ppm} .{ }^{13} \mathrm{C}$ NMR $(75.4 \mathrm{~Hz}$, $\left.\mathrm{CDCl}_{3}\right) \delta_{\mathrm{C}}: 13.20(\mathrm{C}-21), 16.80$ (C-30), 16.90 (C-20), 21.68 (C-47), 22.62 (C-47), 24.00 (C-41), 25.30 (C-15), 26.40 (C-16), 26.80 (C-39),27.46 (C-31),27.82 (C-40),30.30(C-32), 30.90 (C-9),33.33 $(\mathrm{C}-14), 33.66(\mathrm{C}-8), 33.80(\mathrm{C}-3), 34.80$ (C-11),35.82(C-38),38.28(C-6),38.66 (C-1),40.50 (C-36, C-45), 42.80 (C-5), 43.17 (C-13), 44.80 (C2), 51.24 (C-25), 51.26(C-12), 51.62 (C-44), 54.16 (C-26), 56.70 (C-17), 57.34 (C-4), 58.00 (C-35), 69.70 (C-10), 86.46 (C-29), 87.32 (C-28), 133.94 (C-19), 154.08 (C-18), 154.50 (C-33), 156.20 (C42), 162.70 (C-23) ppm. El-MS $m / z$ 646.52 Anal. Calcd. for $\mathrm{C}_{40} \mathrm{H}_{66} \mathrm{~N}_{6} \mathrm{O}: \mathrm{C}, 74.26 ; \mathrm{H}, 10.28 ; \mathrm{N}, 12.99$; $\mathrm{O}, 2.47$. Found: $\mathrm{C}, 74.13 ; \mathrm{H}, 10.14$.

\section{Preparation of 1,4-diazacycloundeca-5,11-dien- steroid derivatives}

A solution of 17 or 19 or $22(0.50 \mathrm{mmol})$, Copper(II) chloride anhydrous (70 mg, $0.52 \mathrm{mmol}$ ), in $5 \mathrm{ml}$ of methanol was stirring for $72 \mathrm{~h}$ to room temperature. The reaction mixture was evaporated to dryness under reduced pressure. After, the residue was purified by crystallization from methanol: water $(3: 1)$.

2-[4-(2-Amino-ethylimino)butyl]-3-[2-(1,4-diazacycloundeca-5,11-dien-5-ylamino)-ethylimino]$5 \mathrm{a}, 7 \mathrm{a}$-dimethyl-2a, 3, 4, 5, 5a, 5 b, 6, 7,7 a, $8,9,10,10 a, 10 b, 11,12$-hexadecahydro-cyclobuta [j]cyclopenta[a]phenanthren-8-ol (18).

yielding $55 \%$ of product, m.p. $116-118^{\circ} \mathrm{C}$; IR $\left(\mathrm{V}_{\max }, \mathrm{cm}^{-1}\right): 3402,3380$ and 3330; ${ }^{1} \mathrm{H}$ NMR (300 $\left.\mathrm{MHz}, \mathrm{CDCl}_{3}\right) \delta_{\mathrm{H}}: 0.84(\mathrm{~s}, 3 \mathrm{H}), 0.94(\mathrm{~m}, 1 \mathrm{H}), 0.98$ $(\mathrm{s}, 3 \mathrm{H}), 1.10-1.64(11 \mathrm{H}), 1.74(\mathrm{~m}, 2 \mathrm{H}), 1.78-1.82(\mathrm{~m}$, $2 \mathrm{H}), 1.84(\mathrm{~m}, 2 \mathrm{H}), 1.86(\mathrm{~m}, 1 \mathrm{H}), 1.88(\mathrm{~m}, 2 \mathrm{H}), 1.98$ $(\mathrm{m}, 1 \mathrm{H}), 2.04(\mathrm{~m}, 2 \mathrm{H}), 2.06(\mathrm{~m}, 2 \mathrm{H}), 2.21-2.24(\mathrm{~m}$, $2 \mathrm{H}), 2.30(\mathrm{~m}, 2 \mathrm{H}), 2.32(\mathrm{~m}, 2 \mathrm{H}), 2.40(\mathrm{~m}, 1 \mathrm{H}), 3.10$ $(\mathrm{m}, 2 \mathrm{H}), 3.12(\mathrm{~m}, 2 \mathrm{H}), 3.48(\mathrm{~m}, 2 \mathrm{H}), 3.52-3.58(\mathrm{~m}$, $4 \mathrm{H}), 3.64(\mathrm{~m}, 1 \mathrm{H}), 3.90(\mathrm{~m}, 2 \mathrm{H}), 4.44(\mathrm{~d}, 1 \mathrm{H}, \mathrm{J}=0.78$ $\mathrm{Hz}), 5.22(\mathrm{~m}, 1 \mathrm{H}), 5.40(\mathrm{~d}, 1 \mathrm{H}, \mathrm{J}=1.32 \mathrm{~Hz}), 5.50$ (broad, $5 \mathrm{H}), 6.70(\mathrm{~m}, 1 \mathrm{H}), 8.20(\mathrm{~m}, 1 \mathrm{H}) \mathrm{ppm} .{ }^{13} \mathrm{C}$ NMR $\left(75.4 \mathrm{~Hz}, \mathrm{CDCl}_{3}\right) \delta_{\mathrm{C}}: 11.80(\mathrm{C}-36), 15.60$ (C35), 21.30 (C-33), 23.74 (C-26), 25.14 (C-32), 25.22
(C-39), 26.64 (C-28), 27.22 (C-7), 27.25 (C-27), 28.88 (C-8), 30.72 (C-25), 31.10 (C-40), 32.06(C31), 32.70 (C-10), 34.12 (C-9), 36.10 (C-38), 36.56 (C-21), 37.30 (C-34), 40.54 (C-44), 42.02 (C-18), 43.60 (C-3), 44.00 (C-23), 45.16 (C-19), 45.87 (C13), 47.40 (C-20), 49.08 (C-17), 51.12 (C-22), 52.72 (C-14), 58.00 (C-43), 61.27 (C-2), 73.34 (C-6), 81.80 (C-24), 132.38 (C-30), 149.60 (C-5), 152.24 (C-41), 156.28 (C-29), 159.88 (C-11), 169.34 (C-16) ppm. El-MS m/z: 618.49 Anal. Calcd. for $\mathrm{C}_{38} \mathrm{H}_{62} \mathrm{~N}_{6} \mathrm{O}: \mathrm{C}$, 73.74; $\mathrm{H}, 10.10 ; \mathrm{N}, 13.58$; O, 2.58. Found: C, 73.62; $\mathrm{H}, 10.02$.

$(1 Z, 5 E)-N-(2-(((Z))-1-((5 a R, 7 a S, 8 S, E)-3-((2-$ (((1E,5Z)-1,4-diazacycloundeca-5,11-dien-5yl)a m ino)ethyl) imino) - 2 - (E) - 4 - ( ( 2 aminoethyl)imino)butyl)-5a,7a-dimethyl$2 a, 3,4,5,5 a, 5 b, 6,7,7 a, 8,9,10,10 a, 10 b, 11,12$ hexadecahydro-cyclobuta [j]cyclopenta[a] phenanthren-8-yl)ethylidene)amino)ethyl)-1,4diazacycloundeca-5,11-dien-5-amine (20).

yielding $66 \%$ of product, m.p. $62-64{ }^{\circ} \mathrm{C}$; IR $\left(\mathrm{V}_{\max }, \mathrm{cm}^{-1}\right)$ : 2402, 3376 and 3330; ${ }^{1} \mathrm{H}$ NMR (300 $\left.\mathrm{MHz}, \mathrm{CDCl}_{3}\right) \delta_{\mathrm{H}}: 0.88(\mathrm{~s}, 3 \mathrm{H}), 0.98(\mathrm{~s}, 3 \mathrm{H}), 1.20-1.58$ $(\mathrm{m}, 10 \mathrm{H}), 1.68(\mathrm{~s}, 3 \mathrm{H}), 1.74(\mathrm{~m}, 2 \mathrm{H}), 1.78-1.82(\mathrm{~m}$, $3 \mathrm{H}), 1.86(\mathrm{~m}, 4 \mathrm{H}), 1.88(\mathrm{~m}, 4 \mathrm{H}), 1.94(\mathrm{~m}, 1 \mathrm{H}) 2.04$ $(\mathrm{m}, 2 \mathrm{H}), 2.06(\mathrm{~m}, 4 \mathrm{H}), 2.12-2.28(\mathrm{~m}, 4 \mathrm{H}), 2.29(\mathrm{~m}$, $2 \mathrm{H}), 2.30(\mathrm{~m}, 4 \mathrm{H}), 2.39-2.40(\mathrm{~m}, 2 \mathrm{H}), 2.80(\mathrm{~m}, 2 \mathrm{H})$ $3.10(\mathrm{~m}, 2 \mathrm{H}), 3.14(\mathrm{~m}, 4 \mathrm{H}), 3.46(\mathrm{~m}, 4 \mathrm{H}), 3.52(\mathrm{~m}$, $2 \mathrm{H})$, 3.56-3.58 $(\mathrm{m}, 4 \mathrm{H}), 3.90(\mathrm{~m}, 2 \mathrm{H}), 3.96-4.42(\mathrm{~m}$, $2 \mathrm{H}), 5.22(\mathrm{~m}, 1 \mathrm{H}), 5.40(\mathrm{~d}, 1 \mathrm{H}, \mathrm{J}=1.82 \mathrm{~Hz}), 5.60$ (broad, 6H), $6.70(\mathrm{~m}, 2 \mathrm{H}), 8.20(\mathrm{~m}, 1 \mathrm{H}) \mathrm{ppm} .{ }^{13} \mathrm{C}$ NMR $\left(75.4 \mathrm{~Hz}, \mathrm{CDCl}_{3}\right) \delta_{\mathrm{C}}: 13.20$ (C-36), 15.60 (C35), 21.92 (C-25), 22.60 (C-61), 25.18 (C-31), 25.22 (C-32), 25.24 (C-38), 26.30 (C-56), 26.40 (C-33), 26.62 (C-27), 26.64 (C-27), 27.20 (C-7, C-59), 27.28 (C-26), 28.84 (C-8, C-8, 31.10 (C-39), 32.04 (C30), 32.70 (C-10), 34.16 (C-9, C-57), 36.14 (C-37), 36.52 (C-21), 38.28 (C-28), 40.56 (C-43), 42.02 (C18), 42.82 (C-23), 43.60 (C-3, C-52), 45.20 (C-19), 45.86 (C-13, C-48), 48.80 (C-20), 49.02 (C-17), 52.22 (C-47), 52.76 (C-14), 54.90 (C-53), 56.20 (C22), 56.70 (C-34), 58.00 (C-42), 61.26 (C-2), 73.40 (C-6, C-60), (C-18), 132.40 (C-29), 149.60 (C-5, C-50), 152.26 (C-40), 156.20 (C-28), 159.90 (C-11, C-55), 162.68 (C-45), 169.28 (C-16) ppm. El-MS $\mathrm{m} / \mathrm{z}$ 836.68 Anal.Calcd.for $\mathrm{C}_{51} \mathrm{H}_{84} \mathrm{~N}_{10}: \mathrm{C}, 73.16 ; \mathrm{H}$, $10.11 ; \mathrm{N}, 16.73$. Found: $\mathrm{C}, 73.08 ; \mathrm{H}, 10.02$. 
10-[5-(2-Aminoethylimino)-penthyl]-3-\{1-[2-(1,4diaza-cycloundeca-5,11-dien-5-ylamino)-ethylimino]ethyl \}-3a, 5b-dimethyl-1, 2, 3, 3a 4, 5, 5a ,5b,6,7,8,9,11a,12,12a,12b-hexadecahydrocyclobuta [k] cyclopenta [a] phenanthren-8-ol (23).

yielding $64 \%$ of product, m.p. $150-152^{\circ} \mathrm{C}$; IR $\left(\mathrm{V}_{\max }, \mathrm{cm}^{-1}\right): 3380$ and 3332; ${ }^{1} \mathrm{H} \mathrm{NMR}(300 \mathrm{MHz}$, $\left.\mathrm{CDCl}_{3}\right) \delta_{\mathrm{H}}: 0.88(\mathrm{~s}, 3 \mathrm{H}), 0.98(\mathrm{~s}, 3 \mathrm{H}), 1.14-1.38(\mathrm{~m}$, $6 \mathrm{H}), 1.40(\mathrm{~m}, 2 \mathrm{H}), 1.42-1.66(\mathrm{~m}, 5 \mathrm{H}), 1.68(\mathrm{~s}, 3 \mathrm{H})$, 1.70-1.84 (m, 4H), 1.86-1.88 (m, 4H), $1.90(\mathrm{~m}, 2 \mathrm{H})$, $1.94(\mathrm{~m}, 1 \mathrm{H}), 2.04(\mathrm{~m}, 2 \mathrm{H}), 2.06-2.10(\mathrm{~m}, 4 \mathrm{H}), 2.12-$ $2.28(\mathrm{~m}, 4 \mathrm{H}), 2.30(\mathrm{~m}, 2 \mathrm{H}), 2.40(\mathrm{~m}, 1 \mathrm{H}), 2.78(\mathrm{~m}$, $2 \mathrm{H}), 3.10(\mathrm{~m}, 2 \mathrm{H}), 3.12(\mathrm{~m}, 2 \mathrm{H}), 3.48(\mathrm{~m}, 2 \mathrm{H}), 3.52$ $(\mathrm{m}, 2 \mathrm{H}), 3.56(\mathrm{~m}, 2 \mathrm{H}), 3.80(\mathrm{~m}, 1 \mathrm{H}), 3.96(\mathrm{~m}, 1 \mathrm{H})$, 4.80 (broad, $5 \mathrm{H}$ ), $5.40(\mathrm{~d}, 1 \mathrm{H}, \mathrm{J}=0.70 \mathrm{~Hz}), 6.70(\mathrm{~m}$, $1 \mathrm{H}), 8.10(\mathrm{~m}, 1 \mathrm{H}) \mathrm{ppm} .{ }^{13} \mathrm{C} \mathrm{NMR}\left(75.4 \mathrm{~Hz}, \mathrm{CDCl}_{3}\right)$ $\delta_{\mathrm{C}}: 13.20$ (C-37), 16.90 (C-38), 21.68 (C-33), 22.62 (C-36), 25.30 (C-31), 26.30 (C-10), 26.40 (C-30), 26.80 (C-41), 27.20 (C-7), 27.82 (C-42), 28.88 (C8), 30.40 (C-43), 30.90 (C-24), 33.33 (C-29), 33.66 (C-23), 33.80 (C-9), 34.12 (C-9), 34.80 (C-26), 35.82 (C-40), 38.28 (C-32), 38.66 (C-22), 40.50 (C-47), 42.80 (C-47), 42.80 (C-18), 43.17 (C-28), 43.60 (C3), 44.80 (C-21), 45.82 (C-13), 51.24 (C-27), 52.26 (C-14), 54.88 (C-2), 56.70 (C-17), 57.34 (C-19), 58.00 (C-46), 69.70 (C-25), 73.40 (C-6), 133.94 (C35), 149.60 (C-5), 154.08 (C-34), 154.50 (C-44), 159.90 (C-11), 162.70 (C-16) ppm.EI-MS m/z 646.52 Anal. Calcd. for $\mathrm{C}_{41} \mathrm{H}_{68} \mathrm{~N}_{6} \mathrm{O}: \mathrm{C}, 74.26 ; \mathrm{H}$, 10.37 ; N, 12.71; O, 2.42. Found: C, 74.14; H, 10.24.

\section{Antimicrobial activity}

The evaluation of antimicrobial effect of the different compounds on the bacterial species was made by the previously method described ${ }^{21}$. The bacterial species were incubated on brain/ heart Infusion (Streptococcus pneumoniae) and Staphylococcus 110 (Staphylococcus aureus) agars for $24 \mathrm{~h}$ at $37^{\circ} \mathrm{C}$. After such time, it was determined whether growth had taken place or not. In addition, a series of tubes were prepared, the first of which contained $2 \mathrm{ml}$ of culture medium (tripticasesoye) at double concentration and the remainder (11tubes), contained the same quantity of medium at single concentrations. From the first tube (double concentration) an aliquot of $2 \mathrm{ml}$ of the studied compound $(1 \mathrm{mg} / \mathrm{ml})$ was added and stirred, from this tube an aliquot of $2 \mathrm{ml}$ was taken and added to the following tube (simple concentration) and the process was successively repeated until the last $2 \mathrm{ml}$ of dissolution had been used up. After this process, each tube was inoculated with $0.1 \mathrm{ml}$ of the bacterial suspension, whose concentration corresponded to Mc-Farland scale $\left(910^{8}\right.$ cells $/ \mathrm{ml}$ ) and all the tubes were incubated at $37{ }^{\circ} \mathrm{C}$ for 24 hours. Subsequently, a loop was taken from each of them and inoculated into the appropriate cultures for different bacterial organisms, and were incubated for $24 \mathrm{~h}$. at $37^{\circ} \mathrm{C}$. After such time, the minimum inhibitory concentration (MIC) was evaluated to consider the antimicrobial effect of the different compounds. In order to discard the effect of methanol (solvent) on the bacterial species studied, a series of the same number of tubes was prepared in parallel, to which $2 \mathrm{ml}$ of methanol at $60 \%$ was added to the first and corresponding successive dilutions were added in the same way as before. In addition a control series was also performed using distilled water to $\mathrm{pH}$ 7.0.

\section{Docking Server}

Docking calculations were carried out using Docking Server ${ }^{22}$. The MMFF94 force field ${ }^{23}$ was used for energy minimization of ligand molecule using the Docking Server. Gasteiger partial charges were added to the ligand atoms. Non-polar hydrogen atoms were merged, and rotatable bonds were defined. Docking calculations were carried out on the HERD2 ${ }^{24}$ and PARP ${ }^{25}$ protein model. Essential hydrogen atoms, Kollman united atom type charges, and solvation parameters were added with the aid of AutoDock tools ${ }^{26}$. Affinity (grid) maps of $20 \times 20 \times 20$-Å grid points and $0.375-\AA$ spacing were generated using the Autogrid program ${ }^{26}$. Auto Dock parameter set and distance dependent dielectric functions were used in the calculation of the Vander Waals and the electrostatic terms, respectively. Docking simulations were performed using the Lamarckian genetic algorithm (LGA) and the Solis and Wets local search method ${ }^{27}$. Initial position, orientation, and torsions of the ligand molecules were set randomly. Each docking experiment was derived from two different runs that were set to terminate after a maximum of 250,000 energy evaluations. The population size was set to 150 . During the search, a translational step of $0.2 \AA$ and quaternion and torsion steps of 5 were applied. 
Evaluation of lipophilicity degree of compounds 18, 20 and 23.

To estimate the logarithmic octanol-water partition coefficient ( $\log \mathrm{P}$ ) of compounds 18, 20 and 23, the logkow method (atom/fragment contribution), introduced by Mannhold and Howard $(\operatorname{method} A)$, available as the KOWWIN and KLOGP (method B) software's and the fragmental technique $\mathrm{ACD} / \log \mathrm{P}$ (method C) were used ${ }^{28}$.

\section{Statistical analysis}

The obtained values are expressed as average \pm SE, using each heart $(n=9)$ as its own control. The data obtained were put under Analysis of Variance (ANOVA) with the Bonferroni correction factor $^{29}$ using the SPSS 12.0 program. The differences were considered significant when $p$ was equal or smaller than 0.05 .

\section{RESULTS AND DISCUSSION}

There are reports which indicate the preparation of diazahicyclo derivatives as antibacterial agents; nevertheless, expensive reagents and special conditions are required ${ }^{9-17}$. Therefore, in this study three diazahicyclo-steroid derivatives were synthetized using several strategies to evaluate the biological activity against Staphylococcus aureus and Streptococcus pneumoniae.

\section{Preparation of threediazecin-steroid-hexahy- droazocin derivatives}

In this study several straightforward routes are reported for synthesis of three diazahicyclosteroid derivatives using OTBS-testosterone (1), progesterone (2) and OTBS-pregnenolone (3) as chemical tools (Scheme 1). The first stage was achieved by the synthesis of three cyclobutanesteroid derivatives ( 4 or 5 or 6 , Scheme 2 ); it is important to mention that there are several reports for preparation of cyclobutene rings using some reagents such as $\mathrm{Co}\left(\mathrm{PPh}_{3}\right)_{2} \mathrm{l}_{2} / \mathrm{PPh}_{3} / \mathrm{Zn}^{30}$, rodhium ${ }^{31}$, nike ${ }^{32}$, ruthenium ${ }^{33}$ and others. In this study, the compounds 1, 2 and 3 were reacted with 5-hexyn1-ol using Cooper II chloride as catalyst to form 4 or 5 or 6 . The ${ }^{1} \mathrm{H}$ NMR spectrum of 4 shows signals at 0.08 and $0.86 \mathrm{ppm}$ for ter-butyldimethylsylane fragment; at 0.68 and 1.08 for methyl groups bound to steroid nucleus; at 0.94-1.04, 1.20-1.48, 1.62-2.10 and $2.22-3.54 \mathrm{ppm}$ for steroid moiety; at 1.52-1.60, 2.20 and $3.66 \mathrm{ppm}$ for methylene groups involved in the arm bound to A-ring; at $5.70 \mathrm{ppm}$ for cyclobutane. The ${ }^{13} \mathrm{C}$ NMR spectra showed chemical shifts at $-4.60,18.02$ and $25.50 \mathrm{ppm}$ for ter-butyldimethylsylane fragment; at 11.32-14.80 ppm for methyl groups bound to steroid nucleus; at 21.34-23.52, 25.24,31.06-32.44,36.54-36.70, $38.50-60.16$ and $81.62 \mathrm{ppm}$ for steroid moiety; at $24.30,32.54,37.02$ and $62.5 \mathrm{ppm}$ for methylene groups involved in the arm bound to A-ring; at $134.52-147.68 \mathrm{ppm}$ for cyclobutane ring; at 210.62 ppm for ketone group. Finally, the presence of 4 was further confirmed from mass spectrum which showed a molecular ion at m/z: 500.36.

On the other hand, the ${ }^{1} \mathrm{H}$ NMR spectrum of 5 showed characteristic signals at 2.12 for methyl group bound to ketone; $3.60 \mathrm{ppm}$ for hydroxyl group; at $5.70 \mathrm{ppm}$ for cyclobutane ring. The ${ }^{13} \mathrm{C}$ NMR spectra showed chemical shifts at $30.74 \mathrm{ppm}$ for methyl group bound to ketone; at 134.52-147.68 ppm for cyclobutane ring; at 208.28-210.62 ppm for ketone groups. In addition, the presence of 5 was further confirmed from mass spectrum which showed a molecular ion at m/z: 412.29.

Finally, the ${ }^{1} \mathrm{H}$ NMR spectrum of 6 shows signals at $1.501 .52,2.02$ and $3.66 \mathrm{ppm}$ for methylene groups involved in the arm bound to A-ring; at $3.60 \mathrm{ppm}$ for hydroxyl group at 5.40 for cyclobutane ring. The ${ }^{13} \mathrm{C}$ NMR spectra showed chemical shifts at $28.34 \mathrm{ppm}$ for methyl group bound to ketone; at 24.90 and $32.54,35.62,62.55$ and $71.56 \mathrm{ppm}$ for methylene groups involved in the arm bound to A-ring; at $133.92-153.90 \mathrm{ppm}$ for cyclobutane ring; at 208.58 ppm for ketone group. Finally, the presence of 6 was further confirmed from mass spectrum which showed a molecular ion at $\mathrm{m} / \mathrm{z}: 528.39$.

\section{Preparation of Cyclobutene-ol-steroid-amino conjugates}

The following stage was achieved by preparation of imino groups involved in the compounds 7 or 8 or 9 (Scheme 2). It is important to mention, that there are several procedures for the synthesis of imino groups which are described in the literature ${ }^{34,35}$. In this study the compounds 7 or 8 or 9 were synthesized (Fig. 3) by the reaction of 3 or 

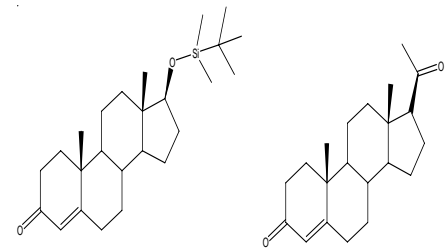

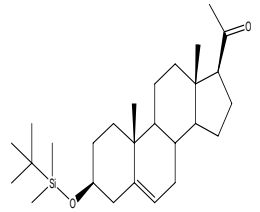

3

Scheme 1. Chemical structure of OTBS-Testosterone (1), Progesterone (2) and OTBS-pregnenolone (3).
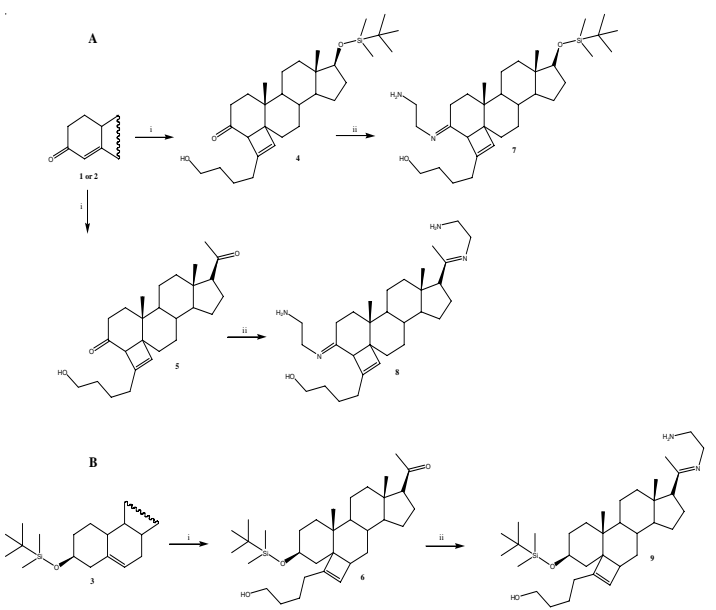

Scheme 2. Preparation of cyclobutene-1-ol-steroidamino conjugates (7 or 8 or 9). Reaction of 1 or 2 or 3 with 5-hexyn-1-ol/Cooper(II) chloride (i) the cyclobuta3-one-steroid derivatives (4 or 5 or 6 ). After, 4 or 5 or 6 were reacted with ethylenediamine/boric acid (ii) to form 7 or 8 or 9 .

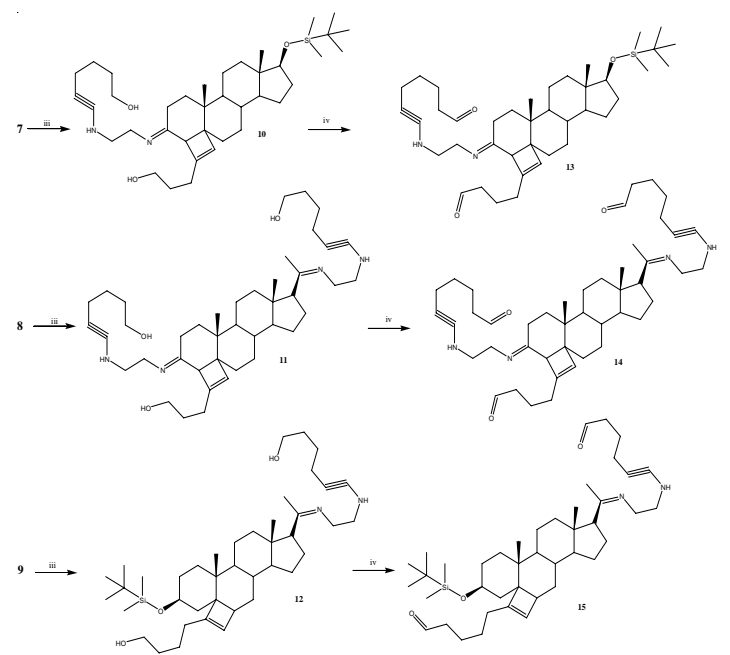

Scheme 3. Preparation of cyclobuta-ynal-steroid derivatives (13 or 14 or 15).Alkynylation of 7 or 8 or 9 with5-hexyn-1-ol/Cooper(II) chloride (iii) to form the heynol-steroid derivatives (10 or 11 or 12). After, 10 or 11 or 12 were reacted with DMSO (iv) to form 13 or 14 or 15.
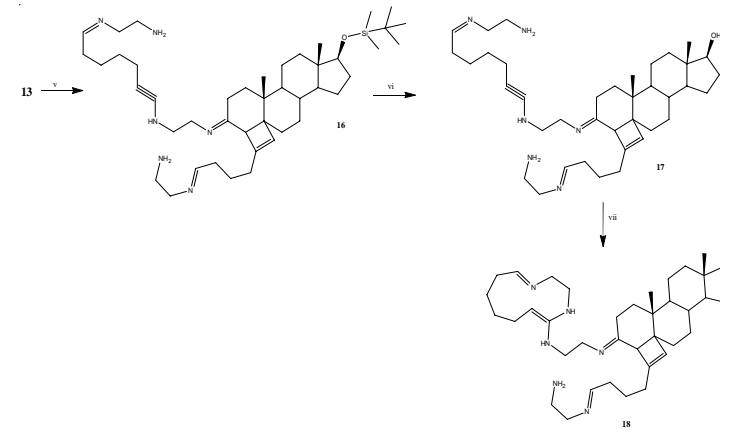

Scheme 4. Synthesis of a diazacycloundeca-5,11-diensteroid-8-ol derivative (18). Reaction of 13 with ethylenediamine (v) to form an amino-steroid derivative (16). After, 17was prepared by removal of the terbuthylsylane fragment of 16 with hydrofluoric acid (v). Finally, 17 was reacted with Copper(II) chloride (vi) to form 18.

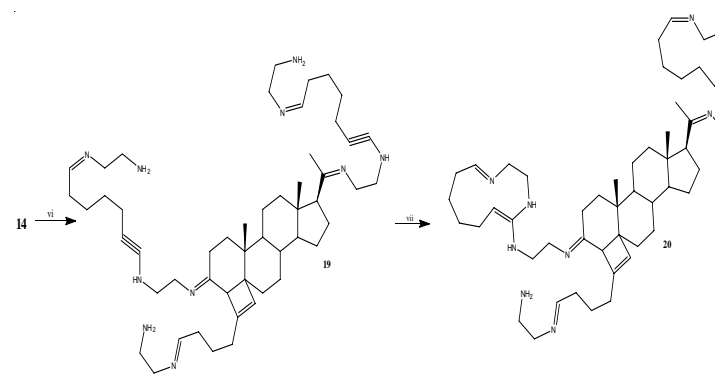

Scheme 5. Preparation of a di-diazacycloundeca-5,11dien-steroid-5-amine derivative (20). Reaction of 14 with ethylenediamine (vi) to form an amino-steroid derivative (19). After, 19 was was reacted with Copper(II) chloride (vi) to form 20.

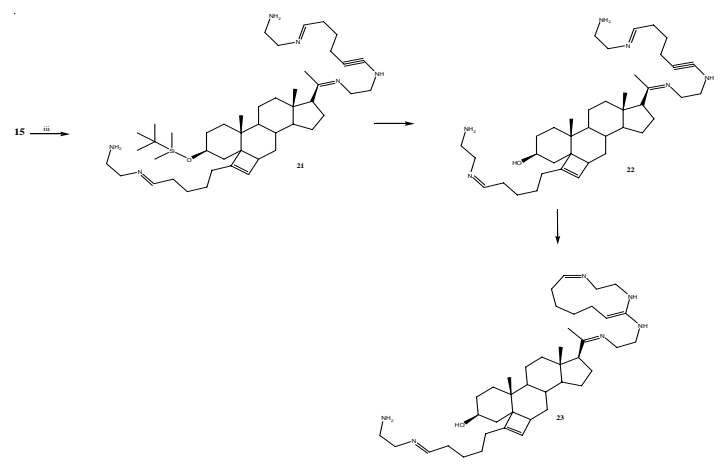

Scheme 6. Synthesis of a diazacycloundeca-5,11-diensteroid-5-amine derivative (23). Reaction of 15 with ethylenediamine (v) to form an amino-steroid derivative (21). After, 22was prepared by removal of the terbuthylsylane fragment of 21 with hydrofluoric acid (v). Finally, 22 was reacted with Copper(II) chloride (vi) to form 23. 
5 or 6 with ethylenediamine using boric acid as catalyst, because it is not an expensive reagent and no special conditions are required for use ${ }^{36}$. The ${ }^{1} \mathrm{H}$ NMR spectrum of 7 showed signals at 1.50, 1.62, 2.15 and $3.66 \mathrm{ppm}$ for methylene groups of arm bound to cylobutene ring; at $3.10-3.50 \mathrm{ppm}$ for methylene groups involved in the arm bound to A-ring of steroid; at $4.10 \mathrm{ppm}$ for both hydroxyl and amino groups; at 5.40 for cyclobutane ring. The ${ }^{13} \mathrm{C}$ NMR spectra showed chemical shifts at 24.38 , 32.54-36.50 and $62.55 \mathrm{ppm}$ for methylene groups of arm bound to cyclobutane ring; at 41.00 and 53.60 ppm for methylene groups involved in the arm bound to A-ring of steroid; at 132.34-150.38 for cyclobutane ring; at $169.34 \mathrm{ppm}$ for imino group. In addition, the presence of 7 was further confirmed from mass spectrum which showed a molecular ion at $\mathrm{m} / \mathrm{z}$ : 542.42.

On the other hand, the ${ }^{1} \mathrm{H}$ NMR spectrum of 8 showed signals at 1.50, 1.62, 2.18 and 3.66 $\mathrm{ppm}$ for methylene groups of arm bound to cyclobutane ring; at 3.09 and $3.52 \mathrm{ppm}$ for methylene groups bound to both amino and imino groups; at 3.10-3.50 ppm for methylene groups involved in the arm bound to A-ring of steroid; at $4.20 \mathrm{ppm}$ for both hydroxyl and amino groups; at $5.40 \mathrm{ppm}$ for cyclobutane ring. The ${ }^{13} \mathrm{C}$ NMR spectra showed chemical shifts at 24.40, 32.54-36.52 and $62.55 \mathrm{ppm}$ for methylene groups of arm bound to cyclobutane ring; at 41.00 and $53.60 \mathrm{ppm}$ for methylene groups involved in the arm bound to Aring of steroid; at 41.02 and $53.10 \mathrm{ppm}$ for methylene groups bound to both amino and imino groups; at $132.32-150.38$ ppm for cyclobutane ring; at 156.78 $169.28 \mathrm{ppm}$ for imino group. In addition, the presence of 8 was further confirmed from mass spectrum which showed a molecular ion at m/z: 496.41 .

Finally, other results showed several signals involved in the ${ }^{1} \mathrm{H}$ NMR spectrum for 9 at; 0.07 and $0.90 \mathrm{ppm}$ for ter-butyldimethylsylane fragment; at 1.50, 1.52 and $2.00 \mathrm{ppm}$ for methylene groups of arm bound to cyclobutane ring; at 3.083.52 and $3.66 \mathrm{ppm}$ for methylene groups involved in the arm bound to A-ring of steroid; at 3.08-3.52 and $3.66 \mathrm{ppm}$ for methylene groups bound to both imino and amino groups; at $4.10 \mathrm{ppm}$ for both hydroxyl and amino groups; at $5.40 \mathrm{ppm}$ for cyclobutane ring. The ${ }^{13} \mathrm{C}$ NMR spectra showed chemical shifts at 29.90, 32.54, 35.57 and 62.55 $\mathrm{ppm}$ for methylene groups of arm bound to cyclobutane ring; at 41.00 and $53.10 \mathrm{ppm}$ for methylene groups bound to both imino and amino groups; at 133.94-153.88 ppm for cyclobutene ring; at $156.70 \mathrm{ppm}$ for imino group. In addition, the presence of 9 was further confirmed from mass spectrum which showed a molecular ion at $\mathrm{m} / \mathrm{z}$ : 570.45 .

\section{Alkynylation of amino groups}

There are some studies which has shown the reaction of chloro-hexyne derivatives with secondary amines; for example, the preparation of $\alpha$-Alkynyl- $\alpha$-amino Esters via the Mannich reaction with silylketeneacetals and alkynyl imines using silver as catalyst ${ }^{37}$. Other data indicate the preparation of an indole-alkyne derivative by the reaction of 5-chloro-1-pentyne or 6-chloro-1-hexyne with indole-3-acetamide in basic medium ${ }^{38}$. In this investigation the compounds 7,8 or 9 were reacted with 5-hexyn-2-ol in presence of Copperll chloride to form 10 or 11 or 12 (Scheme 3). The mechanism involves the compounds 7 or 8 via $\mathrm{SN}_{2}$ mechanism (Fig. 4 and 5). The ${ }^{1} \mathrm{H}$ NMR spectrum of 10 showed signals at 1.58, 1.64, 2.30 and $3.66 \mathrm{ppm}$ for methylene groups bound to both alkyne and hydroxyl groups; at 1.68, 2.26 and $3.52 \mathrm{ppm}$ for methylene groups of arm bound to cyclobutene ring; at 3.20 and $3.56 \mathrm{ppm}$ for methylene groups bound to both amino and imino groups; at $3.80 \mathrm{ppm}$ for both hydroxyl and amino groups; at 5.40 for cyclobutene ring. The ${ }^{13} \mathrm{C}$ NMR spectra showed chemical shifts at 23.14, 33.40 and 61.86 for methylene groups of arm bound to cyclobutene ring; at 51.72 and $54.14 \mathrm{ppm}$ for methylene groups bound to both amino and imino groups; at 78.44 and $87.30 \mathrm{ppm}$ for alkyne group; at 133.52-150.58 ppm for cyclobutene ring; at $169.34 \mathrm{ppm}$ for imino group. Finally, the presence of 10 was further confirmed from mass spectrum which showed a molecular ion at m/z: 624.46 .

On the other hand, the ${ }^{1} \mathrm{H}$ NMR spectrum of 11 showed signals at 1.58-1.66, 2.30 and 3.64 ppm for methylene groups bound to both hydroxyl and alkyne groups; at 1.69, 2.26 and $3.52 \mathrm{ppm}$ for methylene groups involved in the arm bound to 
cyclobutene ring; at 3.16 and $3.56 \mathrm{ppm}$ for methylene groups bound to both amino and imino groups; at $3.70 \mathrm{ppm}$ for both hydroxyl and amino groups; at 5.40 for cyclobutene ring. The ${ }^{13} \mathrm{C}$ NMR spectra showed chemical shifts at 23.20, 33.42 and $61.80 \mathrm{ppm}$ for methylene groups involved in the arm bound to cyclobutene ring; at 51.22-51.76 and $54.10 \mathrm{ppm}$ for methylene groups bound to both amino and imino groups; at 78.48-87.28 ppm for alkyne group at 133.52-150.58 ppm for cyclobutene ring; at 162.68- 169.28 ppm for imino groups. In addition, the presence of 11 was further confirmed from mass spectrum which showed a molecular ion at $\mathrm{m} / \mathrm{z}: 674.51$.

Finally, The ${ }^{1} \mathrm{H}$ NMR spectrum of 12 showed signals at 0.06 and $0.90 \mathrm{ppm}$ for ter-butyldimethylsylane fragment; at 1.52, 2.00 and $3.66 \mathrm{ppm}$ for methylene groups involved in the arm bound to cyclobutene ring; at 1.58, 1.64, 2.30 and $3.67 \mathrm{ppm}$ for methylene groups bound to both hydroxyl and alkyne groups; at 3.18 and 3.54 ppm for methylene groups bound to both amino and imino groups; at $3.58 \mathrm{ppm}$ for both hydroxyl and amino groups; at 5.40 ppm for cyclobutene ring.

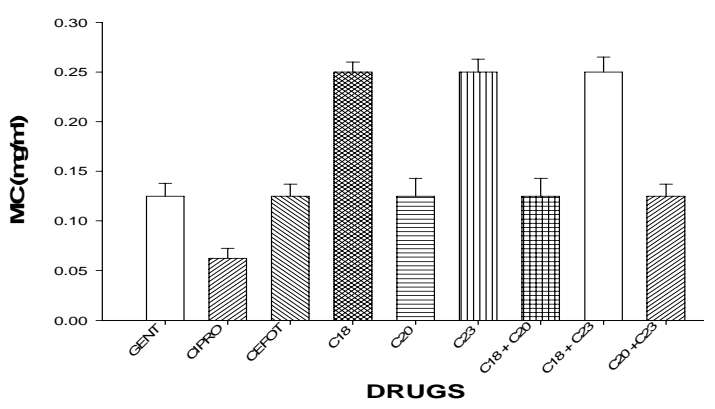

Scheme 7. Antibacterial activity induced by the diazabicyclo-steroid derivatives (compounds C18, C20 and C23) and controls (cefotaxime, CEFOT; gentamicin, GENT; and ciprofloxacin, CIPRO) on Staphylococcus aureus. The results showed that the bacterial growth of Staphylococcus aureuswas inhibited with cefotaxime [MIC $=0.12 \mathrm{mg} / \mathrm{ml}$, gentamicin [MIC $=0.12 \mathrm{mg} / \mathrm{ml}$ ], ciprofloxacin [MIC = $0.06 \mathrm{mg}$ ], compound 18 [MIC $=0.25 \mathrm{mg} / \mathrm{ml}$ ], compound 20 [MIC $=0.12 \mathrm{mg} / \mathrm{ml}$ ], compound 23 [MIC $=0.25 \mathrm{mg} / \mathrm{ml}$. In addition, the growth of this microorganism was inhibited with the following mixtures; $18+20$ [MIC $=0.12 \mathrm{mg} / \mathrm{ml}], 18+23$ [MIC = $0.25 \mathrm{mg} / \mathrm{ml}]$ and $20+23[\mathrm{MIC}=0.12 \mathrm{mg} / \mathrm{ml}]$. MIC = Minimal inhibitory concentration.
The ${ }^{13} \mathrm{C}$ NMR spectra showed chemical shifts at $16.20,25.82,30.10$ and 62.08 ppm for methylene groups bound to both hydroxyl and alkyne groups; at 24.90, 32.54, 35.52 and 62.50 ppm for methylene groups involved in the arm bound to cyclobutene ring; at 51.24 and 56.16 ppm for methylene groups bound to both amino and imino groups; at 78.44$87.32 \mathrm{ppm}$ for alkyne group; at 133.84-153.88 ppm for cyclobutene ring; at $162.70 \mathrm{ppm}$ for imino group. Finally, the presence of 12 was further confirmed from mass spectrum which showed a molecular ion at $\mathrm{m} / \mathrm{z}: 666.51$.

\section{Preparation of aldehyde-steroid derivative.}

The sixth stage was achieved by the synthesis of a aldehyde-steroid derivatives (13 or 14 or 15 , Scheme 3 ); it is noteworthy that there are several reports on the oxidation of primary alcohols to form the corresponding aldehydes. In addition, some reports indicate the preparation of aldehyde derivatives using several reagents such as morpholinium bisulfate ${ }^{39}$, calcium hydride ${ }^{40}$, 2-(hydroxyalky1)dithianes ${ }^{41}, \mathrm{KN}(\mathrm{TMS})_{2}^{42}$, chromium $(\mathrm{VI})^{43}$,

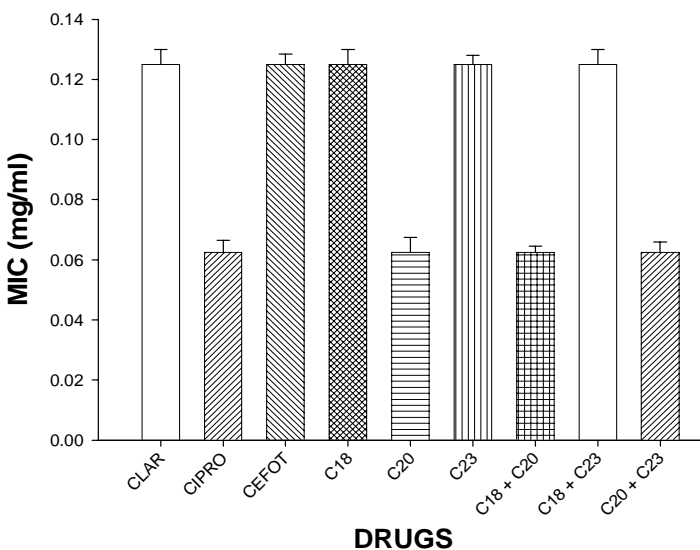

Scheme 8. Effect exerted by the diazabicyclo-steroid derivatives (compounds $\mathrm{C} 18, \mathrm{C} 20$ and $\mathrm{C} 23$ ) and controls (cefotaxime, CEFOT; clarithromicin, CLAR; and ciprofloxacin, CIPRO) on Streptococcus pneumoniae. The results showed that bacterial growth of Streptococcus pneumoniae was inhibited in the presence of cefotaxime [MIC $=0.12 \mathrm{mg} / \mathrm{ml}, 2.74$ ), ciprofloxacin [MIC $=0.06 \mathrm{mg} / \mathrm{ml}$, clarithromycin[MIC $=0.12 \mathrm{mg} / \mathrm{ml}]$ and compounds 18 [MIC $=0.12 \mathrm{mg} / \mathrm{ml}], 20[\mathrm{MIC}=0.0 .06 \mathrm{mg} /$ $\mathrm{ml}]$ and 23 [MIC $=0.12 \mathrm{mg} / \mathrm{ml}$ ]. Additionally, the bacterial growth of Streptococcus pneumoniae was inhibited with the following mixtures; $18+20$ [MIC $=0.06 \mathrm{mg} / \mathrm{ml}], 18+23$ $[M I C=0.12 \mathrm{mg} / \mathrm{ml}], 20+23[\mathrm{MIC}=0.06 \mathrm{mg} / \mathrm{ml}] . \mathrm{MIC}=$ Minimal inhibitory concentration. 
ruthenium ${ }^{44}$ and others. However, some these reagents may induce risks of toxicity by generation of several substances involved on the reaction mixtures. Therefore, in this study a method previously reported ${ }^{37}$ for oxidation of hydroxyl groups was used for synthesis of 13 or 14 or 15 by the reaction of 10 or 11 or 12 with dimethyl sulfoxide. The ${ }^{1} \mathrm{H}$ NMR spectrum of 13 shows signals at 1.70 , $1.84,2.24$ and $2.56 \mathrm{ppm}$ for methylene groups bound to both aldehyde and alkyne groups; at 1.94 and 2.26-2.30 ppm for methylene groups involved in the arm bound to both aldehyde and cyclobutene ring; at 3.20 and 3.56 for methylene groups bound to both amino and imino groups; at $5.20 \mathrm{ppm}$ amino group; at $5.40 \mathrm{ppm}$ for cyclobutene ring; at 9.70$9.82 \mathrm{ppm}$ for aldehyde groups. The ${ }^{13} \mathrm{C}$ NMR spectra showed chemical shifts at 16.18, 22.14, 26.94 and $44.52 \mathrm{ppm}$ for methylene groups involved in the arm bound to both aldehyde and cyclobutene ring; at 51.72 and 54.14 for methylene groups bound to both amino and imino groups; at 79.64 and 87.30 ppm for alkyne group; at 132.38-147.80 for cyclobutene ring; at $169.34 \mathrm{ppm}$ for imino group; at 202.18-202.44 for aldehyde groups. In addition, the presence of 13 was further confirmed from mass spectrum which showed a molecular ion at $\mathrm{m} / \mathrm{z}$ : 666.51 .

Other signals of ${ }^{1} \mathrm{H}$ NMR spectrum for 14 were found at $1.68 \mathrm{ppm}$ for methyl group bound to imino group; at 1.70, 1.86, 2.24 and $2.56 \mathrm{ppm}$ for methylene groups bound to both aldehyde and alkyne groups; at 1.96, 2.26 and $2.30 \mathrm{ppm}$ for methylene groups involved in the arm bound to both aldehyde and cyclobutene ring; at 3.16 and 3.56 ppm for methylene groups bound to both amino and imino groups; at $5.20 \mathrm{ppm}$ for amino group; at $5.40 \mathrm{ppm}$ for cyclobutene group; at 9.70-9.82 ppm for aldehyde groups. The ${ }^{13} \mathrm{C}$ NMR spectra showed chemical shifts at 16.12, 22.10 and $26.90 \mathrm{ppm}$ for methylene groups bound to both alkyne and aldehyde groups; at 21.00, 35.54, 43.50 and 44.62 ppm for methylene groups involved in the arm bound to both aldehyde and cyclobutene ring; at 51.22-51.76 and $54.10 \mathrm{ppm}$ for methylene groups bound to amino and imino groups; at 79.60-87.28 ppm for alkyne group; at 132.40-147.80 ppm for cyclobutene ring; at $162.68-169.28 \mathrm{ppm}$ for imino groups; at 202.20-202.42 ppm for aldehyde groups.
Finally, the presence of 14 was further confirmed from mass spectrum which showed a molecular ion at $\mathrm{m} / \mathrm{z}: 710.51$.

Other results indicated that ${ }^{1} \mathrm{H}$ NMR spectrum of 15 shows signals at 1.84 and 2.44-2.45 ppm for methylene groups bound to both alkyne and aldehyde groups; at 1.46 and 1.56, 1.96 and $2.34 \mathrm{ppm}$ for methylene groups involved in the arm bound to both aldehyde and cyclobutene ring; at 3.18 and 3.56 for methylene groups bound to both amino and imino groups; at $5.20 \mathrm{ppm}$ for amino group; at 5.40 for cyclobutene ring; at $9.66-9.72 \mathrm{ppm}$ for aldehyde groups. The ${ }^{13} \mathrm{C}$ NMR spectra showed chemical shifts at 15.20, 21.72 and $43.12 \mathrm{ppm}$ for methylene groups bound to both alkyne and aldehyde groups; at 23.00-25.30, 35.52 and 43.69 ppm for methylene groups involved in the arm bound to both aldehyde and cyclobutene ring; at 51.26 and $54.16 \mathrm{ppm}$ for methylene groups bound to both amino and imino groups; at 133.90-154.08 ppm for cyclobutene ring; at $162.70 \mathrm{ppm}$ for imino group; at 198.62-202.48 ppm for aldehyde groups. Finally, the presence of 15 was further confirmed from mass spectrum which showed a molecular ion at $\mathrm{m} / \mathrm{z}$ : 666.51.

\section{Preparation of imino-steroid derivatives (Scheme 4, 5 and 6)}

The compounds 13 or 14 or 15 were reacted with ethylenediamine to form the compounds 16 or 19 or 21 using boric acid as catalyst. The ${ }^{1} \mathrm{H}$ NMR spectrum of 16 showed several signals at 0.08 and $0.84 \mathrm{ppm}$ for ter-butyldimethylsylane fragment; at 1.58, 1.64, 2.20 and $2.36 \mathrm{ppm}$ for methylene bound to both amino and alkyne groups; at 1.74, 2.04 and 2.28 ppm for methylene groups bound to both imino and cyclobutene ring; at 3.10, 3.18, 3.52 and $3.56 \mathrm{ppm}$ for methylene groups bound to both imino and amino groups; at $4.52 \mathrm{ppm}$ for amino groups; at 5.40 for proton of cyclobutene ring; at 7.70 and $8.20 \mathrm{ppm}$ for imino groups. The ${ }^{13} \mathrm{C}$ NMR spectra showed chemical shifts at 16.38, 24.92, 29.94 and $27.40 \mathrm{ppm}$ for methylene groups bound to both imino and alkyne; at 25.22, 31.10 and 36.10 ppm for methylene groups bound to both imino and cyclobutene ring; at 40.54, 51.62-51.70 and 54.14$58.00 \mathrm{ppm}$ for methylene bound to both imino and amino groups; 79.64 and $87.30 \mathrm{ppm}$ for alkyne group; at 132.38 and 156.22 ppm for cyclobutene 
ring; at 152.24-156.18 and $169.34 \mathrm{ppm}$ for imino groups. Finally, the presence of 16 was further confirmed from mass spectrum which showed a molecular ion at m/z: 732.58 .

Other data showed several signals of the ${ }^{1} \mathrm{H}$ NMR spectrum for 19 at 1.58, 1.66, 2.20 and $2.36 \mathrm{ppm}$ for methylene groups bound to both imino and alkyne; at 2.06 and $2.30 \mathrm{ppm}$ for methylene groups bound to both imino and cyclobutene ring; at $3.10,3.18,3.52$ and $3.56 \mathrm{ppm}$ for methylene groups bound to both amino and imino groups; at $4.60 \mathrm{ppm}$ for amino groups; at $5.40 \mathrm{ppm}$ for cyclobutene ring; at 7.70-8.20 ppm for imino groups. The ${ }^{13} \mathrm{C}$ NMR spectra showed chemical shifts at 16.42, 24.90, 27.00 and 27.44 ppm for methylene groups bound to both alkyne and imino groups; at 25.22, 31.10 and 36.14 ppm for methylene groups bound to both imino and cyclobutene ring; at 40.56 , 51.22-54.10 and 58.00 ppm for methylene groups bound to both imino and amino groups; at 79.60-87.28 for alkyne groups; at 132.40 and 156.28 for cyclobutene ring; at 152.26, 156.20, 162.68 and $169.28 \mathrm{ppm}$ for imino groups. In addition, the presence of 19 was further confirmed from mass spectrum which showed a molecular ion at $\mathrm{m} / \mathrm{z}$ : 836.68 .

Finally, the ${ }^{1} \mathrm{H}$ NMR spectrum of 21 shows signals at $1.40,1.90$ and 2.06-2.10 ppm for methylene groups bound to both imino and cyclobutene ring; at $1.76,2.12$ and 2.42 ppm for methylene bound to both alkyne and imino groups; at 3.10-3.52 ppm for methylene groups bound to both imino and amino groups; at $4.52 \mathrm{ppm}$ for cyclobutene ring; at $8.10 \mathrm{ppm}$ for imino groups. The ${ }^{13} \mathrm{C}$ NMR spectra showed chemical shifts at 16.80 , 27.46 and 30.30 ppm for methylene groups bound to both alkyne and imino groups; at 26.80, 27.82, 30.40 and 35.82 ppm for methylene groups bound to both imino and cyclobutene ring; at 40.50 , 51.26-54.16 and 58.00 ppm for methylene groups bound to both amino and imino groups; at 86.46-87.32 ppm for alkyne groups; at 133.94154.08 ppm for cyclobutene ring; at 154.50-167.70 ppm for imino groups. Finally, the presence of 21 was further confirmed from mass spectrum which showed a molecular ion at m/z: 760.61.

\section{Removal of silyl fragment of 16 or 22 via hydrofluoric acid to form 17 or 21 (Scheme 4 and 5).}

There are several reagent for removal of silyl protecting groups from hydroxyl such as ammonium fluoride ${ }^{45}$, tris(dimethylamino)sulfonium /difluorotrimethyl silicate ${ }^{46}$, hydrofluoric acid $^{47}$ and others. In this study, hydrofluoric acid was used to removal of silyl-protecting group from hydroxyl of the compound 8 to form 9 (Scheme 5). The ${ }^{1} \mathrm{H}$ NMR spectrum of 17 showed several signals at $1.58,1.66$, 2.20 and $2.36 \mathrm{ppm}$ for methylene bound to both amino and alkyne groups; at 1.74, 2.04 and 2.28 ppm for methylene groups bound to both imino and cyclobutene ring; at 3.10, 3.18 and 3.52-3.56 ppm for methylene groups bound to both imino and amino groups; at $4.80 \mathrm{ppm}$ for both hydroxyl and amino groups; at 5.40 for proton of cyclobutene ring; at 7.70 and $8.20 \mathrm{ppm}$ for imino groups. The ${ }^{13} \mathrm{C}$ NMR spectra showed chemical shifts at 16.38 , 24.92, 26.94 and 27.40 ppm for methylene groups bound to both imino and alkyne; at 25.22, 31.10 and $36.10 \mathrm{ppm}$ for methylene groups bound to both imino and cyclobutene ring; at 40.54, 51.62-58.00 ppm for methylene bound to both imino and amino groups; at 79.64-87.30 ppm for alkyne groups; at 132.38 and $156.22 \mathrm{ppm}$ for cyclobutene ring; at 152.24-156.18 and 169.34 ppm for imino groups. Finally, the presence of 17 was further confirmed from mass spectrum which showed a molecular ion at m/z: 618.49.

Other results showed several signals of the ${ }^{1} \mathrm{H}$ NMR spectrum for 22 at 0.88 and $0.98 \mathrm{ppm}$ for methyl bound to steroid nucleus; at $1.68 \mathrm{ppm}$ for methyl group bound to imino group; at 1.40, 1.90 and 2.06-2.10 ppm for methylene groups bound to both imino and cyclobutene ring; at 1.76, 2.12 and $2.42 \mathrm{ppm}$ for methylene bound to both alkyne and imino groups; at 3.10-3.56 ppm for methylene groups bound to both imino and amino groups; at $4.24 \mathrm{ppm}$ for both hydroxyl and amino groups; at $6.00 \mathrm{ppm}$ for cyclobutene ring; at 7.70-8.10 ppm for imino groups. The ${ }^{13} \mathrm{C}$ NMR spectra showed chemical shifts at $16.80,26.80,27.46$ and 35.82 ppm for methylene groups bound to both alkyne and imino groups; at 40.50, 51.24, 51.62-54.16 and $58.00 \mathrm{ppm}$ for methylene groups bound to both imino and cyclobutene ring; at 40.50, 51.26-54.16 
and $58.00 \mathrm{ppm}$ for methylene groups bound to both amino and imino groups; at $86.46-87.32 \mathrm{ppm}$ for alkyne groups; at 133.94-154.08 ppm for cyclobutene ring; at $154.50-162.70 \mathrm{ppm}$ for imino groups. Finally, the presence of 22 was further confirmed from mass spectrum which showed a molecular ion at $\mathrm{m} / \mathrm{z}: 646.52$.

Synthesis of 1,4-diazacycloundeca-5,11-diensteroid derivatives (Scheme 4, 5 and 6).

Thre are several reports shown the preparation of diazabicycle derivatives using different reagents such as piperidinone ${ }^{48}$, lithium hexamethyldisilazane ${ }^{49}$, sodium hydride ${ }^{50}$, azodicarboxylate ${ }^{51}$, di-tertbutyldicarbonate ${ }^{52}$, palladium on carbon ${ }^{53}$. In this study, the compounds 17, 19 or 22 were reacted with Copper (II) chloride to form the bicycle derivatives 18 or 20 or 23 . The ${ }^{1} \mathrm{H}$ NMR spectrum of 18 showed several signals at $1.74,2.04$ and $2.30 \mathrm{ppm}$ for methylene groups bound to both imino and cyclobutene ring; at 3.10 and 3.52-3.58 ppm for methylene groups bound to both imino and amino groups; at 1.84, 1.88, 2.06, 2.32, 3.12 and $3.90-4.40 \mathrm{ppm}$ for bicycle ring (1,4-diaza-cycloundeca-diene); at $5.40 \mathrm{ppm}$ for cyclobutene ring; at $5.50 \mathrm{ppm}$ for amino groups; at 6.70-8.20 ppm for imino groups. The ${ }^{13} \mathrm{C}$ NMR spectra showed chemical shifts at 25.22, 31.10 and $36.10 \mathrm{ppm}$ for methylene groups bound to both imino and cyclobutene ring; at 40.54, 45.87 and 52.72-58.00 ppm for methylene bound to both imino and amino groups; at 27.22, 28.88, 32.70-34.12, $43.60,61.27-73.34$ and 149.60 for bicycle ring (1,4-diaza-cycloundeca-diene); 132.38 and 156.28 ppm for cyclobutene ring; at 152.24, 159.88-169.34 ppm for imino groups. In addition, the presence of 18 was further confirmed from mass spectrum which showed a molecular ion at m/z: 618.49.

Other data showed several signals of the ${ }^{1} \mathrm{H}$ NMR spectrum for 20 at $1.74,2.04$ and $2.29 \mathrm{ppm}$ for methylene groups bound to both imino and cyclobutene ring; at 1.86-1.88, 2.06, 2.30, 2.80, 3.14 and 3.90-4.42 ppm for bicycle ring (1,4-diazacycloundeca-diene); at 3.10 and 3.46-3.58 ppm for methylene groups bound to both amino and imino groups; at $5.60 \mathrm{ppm}$ for amino groups; at $5.40 \mathrm{ppm}$ for cyclobutene ring; at $6.70-8.20 \mathrm{ppm}$ for imino groups. The ${ }^{13} \mathrm{C}$ NMR spectra showed chemical shifts at $25.24,31.14$ and 36.14 ppm for methylene groups bound to both imino and cyclobutene ring; at $26.30,27.20,28.84,32.70-34.16,43.60,54.90$, 61.26-73.40 and $149.60 \mathrm{ppm}$ for bicycle ring (1,4-diaza-cycloundeca-diene); at 36.14, 40.56, $45.86,52.22-52.76$ and 58.00 ppm for methylene groups bound to both imino and amino groups; at 132.40 and 156.20 for cyclobutene ring; at 152.26 , 159.90-169.28 ppm for imino groups. In addition, the presence of 20 was further confirmed from mass spectrum which showed a molecular ion at $\mathrm{m} / \mathrm{z}$ : 836.68.

Finally, other data showed several signals of the ${ }^{1} \mathrm{H}$ NMR spectrum for 23 at 1.40, 1.90 and 2.06-2.10 ppm for methylene groups bound to both imino and cyclobutene ring; at 1.86-1.88, 2.04, 2.30, $2.78,3.12$ and $3.96 \mathrm{ppm}$ for bicycle ring (1,4-diaza-cycloundeca-diene); at 3.10-3.56 ppm for methylene groups bound to both imino and amino groups; at $4.80 \mathrm{ppm}$ for both hydroxyl and amino groups; at $5.40 \mathrm{ppm}$ for cyclobutene ring; at 6.70$8.10 \mathrm{ppm}$ for imino groups. The ${ }^{13} \mathrm{C}$ NMR spectra showed chemical shifts at 26.30, 27.20, 28.88, $34.12,43.60,54.88,73.40$ and $149.57 \mathrm{ppm}$ for bicycle ring (1,4-diaza-cycloundeca-diene); at $26.80,27.82,30.40,35.82,54.88$ and 154.50 ppm for methylene groups bound to both imino and cyclobutene ring; at 40.50, 45.82, 52.24 and 58.00 ppm for methylene groups bound to both amino and imino groups; at $133.94-154.08 \mathrm{ppm}$ for cyclobutene ring; at 159.90-162.70 ppm for imino groups. Finally, the presence of 23 was further confirmed from mass spectrum which showed a molecular ion at m/z: 646.52.

\section{Evaluation of biological activity for steroid derivatives}

In this study the antibacterial activity of some steroid derivatives (compounds 1-23) on Staphylococcus aureusand Streptococcus pneumoniae was evaluated by means of the dilution method and the minimum inhibitory concentration [MIC], using gentamicin, clarithromycin, ciprofloxacin and cefotaxime as control. The results showed that the bacterial growth of Staphylococcus aureuswas inhibited with cefotaxime [MIC $=0.12$ $\mathrm{mg} / \mathrm{ml}$ ], gentamicin [MIC $=0.12 \mathrm{mg} / \mathrm{ml}$ ], ciprofloxacin [MIC $=0.06 \mathrm{mg}]$, compound $18[\mathrm{MIC}=0.25 \mathrm{mg} / \mathrm{ml}]$, compound $20[\mathrm{MIC}=0.12 \mathrm{mg} / \mathrm{ml}]$, compound 23 
$[\mathrm{MIC}=0.25 \mathrm{mg} / \mathrm{ml}]$. In addition, the growth of this microorganism was inhibited with the following mixtures; $18+20[\mathrm{MIC}=0.12 \mathrm{mg} / \mathrm{ml}], 18+23[\mathrm{MIC}$ $=0.25 \mathrm{mg} / \mathrm{ml}]$ and $20+23[\mathrm{MIC}=0.12 \mathrm{mg} / \mathrm{ml}]$.

Other results showed that bacterial growth of Streptococcus pneumoniae was inhibited in the presence of cefotaxime [MIC $=0.12 \mathrm{mg} / \mathrm{ml}, 2.74$ ), ciprofloxacin $[\mathrm{MIC}=0.06 \mathrm{mg} / \mathrm{ml}$, clarithromycin $[\mathrm{MIC}=0.12 \mathrm{mg} / \mathrm{ml}]$ and compounds $18[\mathrm{MIC}=0.12$ $\mathrm{mg} / \mathrm{ml}$, compound 17 [MIC $=0.06 \mathrm{mg} / \mathrm{ml}], 20$ [MIC $=0.12 \mathrm{mg} / \mathrm{ml}]$ and $23[\mathrm{MIC}=0.12 \mathrm{mg} / \mathrm{ml}]$. Finally, the bacterial growth of Streptococcus pneumoniae was inhibited with the following mixtures; $18+20$ $[\mathrm{MIC}=0.06 \mathrm{mg} / \mathrm{ml}], 18+23[\mathrm{MIC}=0.12 \mathrm{mg} / \mathrm{ml}], 20$ $+23[\mathrm{MIC}=0.06 \mathrm{mg} / \mathrm{ml}$. All these data indicate that; i)compounds 18, 20 and 22 has different antibacterial potency for Staphylococcus aureusand Streptococcus pneumoniae in comparison with gentamicin (an inhibitor of protein synthesis) ${ }^{54}$, and clarithromycin (protein synthesis inhibitor $)^{54}$, this phenomenon may be attributed mainly to the different molecular mechanism involved and the characteristic chemical structure of the compounds studied; ii) the compound 20 exerts greater antibacterial activity against
Staphylococcus aureus and Streptococcus pneumoniae compared with the compounds 18 and 23; iv) the antibacterial effect of 20 was similar to cycprofloxacin. This phenomenon could depend on the interaction of two diazabicyclo rings involved in the chemical structure of 20 with some cellular structure involved in the microorganisms studied. This hypothesis can be availed by some reports which indicate that antibacterial activity of cycprofloxacin is via interaction with DNA gyrase ${ }^{55}$; v) finally, the different mixtures evaluated in this study do not increase the antibacterial activity compared with the compound 20 against Staphylococcus aureus and Streptococcus pneumoniae.

\section{Docking evaluation}

In order, to evaluate the possibility that the compound 20 could interact with DNA gyrase (PDB ID:2xcr $)^{56}$ in this study a molecular docking model (serverdoking) ${ }^{57}$ was used ${ }^{58,59}$. Theoretical results indicate that hydrogen-interaction between compound 20 and DNA gyrase (Fig. 11, 12 and Table 1)involves several amino acid residues such as Leu ${ }_{704}$, Asn $_{705}$, Met $_{780}$, Cys $_{784}$, Met $_{749}$, Leu $_{762}$, Phe $_{764}$, Ser $_{778}$ and Met $_{787}$. In addition,other theoretical results
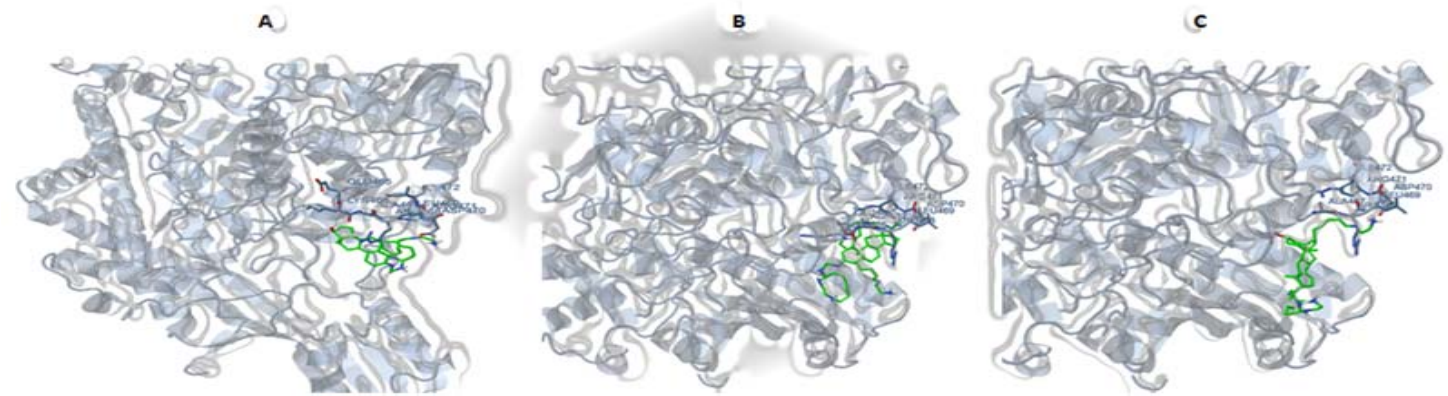

Schme 9. Site of binding for DNA-gyrase with the compounds 18 (A), 20(B) and 23 (C). Visualized with GLmolViewer after docking analysis with one-click docking
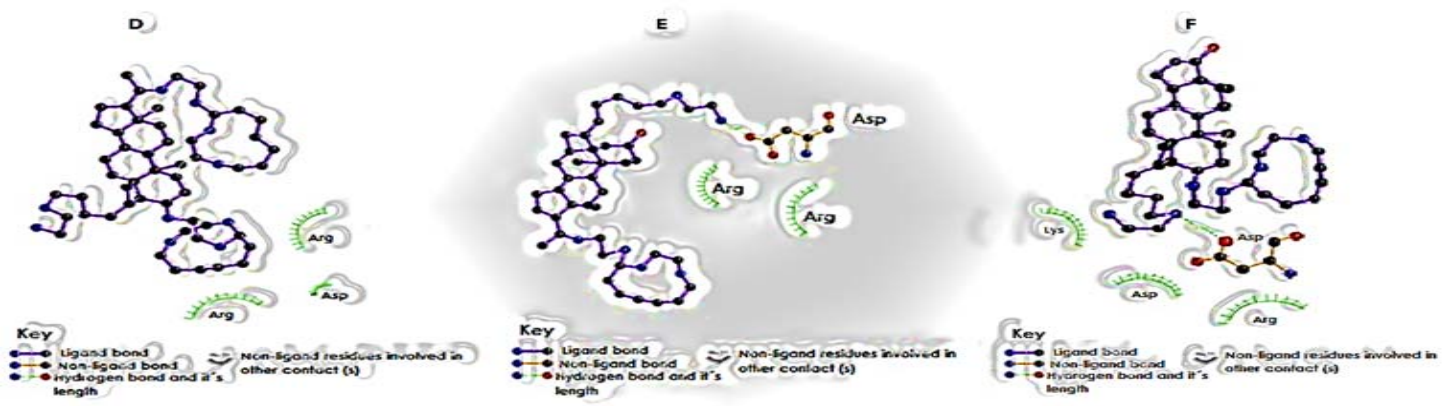

Scheme 10. The scheme shown the contact site of amino acid residues involved in the interaction of DNA-gyrase with the compound 18 (D), 20 (E) and 23 (F). Visualized with GL mol Viewer after docking analysis with one-click docking 
Table: 1. Aminoacid residues involved between the interaction of diazabicyclo-steroid derivatives $(18,20$ and 23$)$ with the DNAgyrase surface

\begin{tabular}{lccc}
\hline \multicolumn{4}{c}{ Compounds } \\
\hline Interactions & $\mathbf{1 8}$ & $\mathbf{2 0}$ & $\mathbf{2 3}$ \\
\hline Hidrogen bonds & $\begin{array}{l}\mathrm{Asp}_{470} \\
\mathrm{Asp}_{470}\end{array}$ & & $\mathrm{Arg}_{470}$ \\
Polar & $\mathrm{Arg}_{471}$ & $\mathrm{Arg}_{471}$ & $\mathrm{Arg}_{468}$ \\
& $\mathrm{Lys}_{466}$ & $\mathrm{Arg}_{468}$ & $\mathrm{Arg}_{470}$ \\
Others & $\mathrm{Asp}_{470}$ & $\mathrm{Asp}_{470}$ & $\mathrm{Arg}_{468}$ \\
& $\mathrm{Arg}_{471}$ & $\mathrm{Arg}_{471}$ & $\operatorname{Arg}_{477}$ \\
\hline
\end{tabular}

*aminoacids residues

showed the decomposed interaction energies (Kcal/ $\mathrm{mol}$ ) between the compound 20 and the amino acid residues from DNA gyrase (table 2). All these data suggest that the interaction of compound 20 with DNA gyrase is conditioned by their physicochemical properties.

Evaluation lipophilicity degree of compounds 18, 20 and 23.
In order to delineate the structural chemical requirements of compounds 18, 20 and 23; other parameters such as the $\log P$ and $\pi$ descriptors were calculated, with the purpose to know if there are differences in its lipophilicity degree ${ }^{60}$. There are studies which indicate that $\log P$ estimates the logarithmic octanol-water partition coefficient; therefore the logPrepresents the lipophilic effects of a molecule which includes the sum of the lipophilic contributions of the parent molecule and its substituent ${ }^{61}$. The difference between the substituted and unsubstituted $\log P$ values is conditioned by the $\partial$ value for the particular substituent. Hammett showed that $ð$ values measure the free energy change caused by particular substituent to relate to biological activity ${ }^{62,63}$.

Analyzing this data, in this study the $\log P$ and $\pi$ parameters were calculated by the method proposed by Mannhold and Waterbeemd ${ }^{64}$. The results (Table 4) showed an increase in both $\log P$ and $\pi$ values of 20 with respect to the compounds 18 and 23 which is mainly conditioned by the contribution of all the substituent atoms involved in the chemical structure of compound 20. This phenomenon conditions the interaction of compound 20 with the DNA gyrase and

Table: 2. Descompesed interaction energies (Kcal/mol) involved between the diazabicyclosteroid derivatives $(18,20$ and 23$)$ and DNA-gyrase surface

\begin{tabular}{lccc}
\hline \multicolumn{4}{c}{ Compounds } \\
\hline Interactions* & $\mathbf{1 8}$ & $\mathbf{2 0}$ & $\mathbf{2 3}$ \\
\hline Hidrogen bonds & $\operatorname{Asp}_{470}(-0.3491)$ & & $\operatorname{Arg}_{470}(0.0708)$ \\
Polar & $\operatorname{Arg}_{471}(-1.4456)$ & $\operatorname{Arg}_{471}(-1.6256)$ & $\operatorname{Arg}_{468}(-1.2042)$ \\
Others & $\operatorname{Arg}_{468}(-1.0132)$ & $\operatorname{Arg}_{468}(-2.2648)$ & $\operatorname{Arg}_{471}(-0.8197)$ \\
& $\operatorname{Lys}_{466}(-0.9578)$ & $\operatorname{Asp}_{470}(-0.1188)$ & \\
\hline
\end{tabular}

*aminoacids residues

Table: 3. Estimated docking parameters involved between the interaction of diazabicyclo-steroid derivatives (18, 20 and 23) with the DNA-gyrase surface

\begin{tabular}{lccccc}
\hline Compounds & $\begin{array}{c}\text { Est. free energy of } \\
\text { binding (kcla/mol) }\end{array}$ & $\begin{array}{c}\text { WW + H bond } \\
\text { + desolv energy }\end{array}$ & $\begin{array}{c}\text { Electrostatic } \\
\text { energy }\end{array}$ & $\begin{array}{c}\text { Interaction } \\
\text { surface }\end{array}$ & Total \\
\hline 18 & -3.80 & -3.80 & -0.87 & 498.002 & -4.67 \\
20 & 2.34 & -3.55 & 0.53 & 543.180 & -3.02 \\
23 & -0.33 & -2.70 & -1.64 & 397.296 & -4.33 \\
\hline
\end{tabular}


Table: 4. Physicochemical parameters [ $\log P(\log K o w)$, and $\pi]$ of compounds 18, 20 and 23

Compound 18

Compound 20

Compound 23
$-\mathrm{CH}_{3} \quad$ [aliphatic carbon]

$-\mathrm{CH}_{2}-$ [aliphatic carbon]

$-\mathrm{CH}^{2}$ [aliphatic carbon]

C [aliphatic carbon- No $\mathrm{H}$, not tert]

$=\mathrm{CH}-$ or $=\mathrm{C}<$ [olefinic carbon]

$-\mathrm{OH}$ [hydroxyl, aliphatic attach]

$-\mathrm{NH}_{2} \quad$ [aliphatic attach]

$-\mathrm{NH}^{2} \quad$ [aliphatic attach]

-tert Carbon [3 or more carbon attach]

$-\mathrm{N}=\mathrm{C} \quad$ [aliphatic attach]

Fused aliphatic ring unit correction

$>\mathrm{C}=\mathrm{N}-\mathrm{C}$ [cyclic-type imine, ali carbon attach]

$\mathrm{C}=\mathrm{C}(-\mathrm{N}-)-\mathrm{N}$ - correction

$-\mathrm{CH}=\mathrm{N}-\mathrm{C}$ [linear imine] correction

Equation Constant

Log Kow

$-\mathrm{CH}_{3} \quad$ [aliphatic carbon]

$-\mathrm{CH}_{2}-$ [aliphatic carbon]

$-\mathrm{CH}$ [aliphatic carbon]

C [aliphatic carbon- No $\mathrm{H}$, not tert]

$=\mathrm{CH}$ - or $=\mathrm{C}<$ [olefinic carbon]

$-\mathrm{NH}_{2} \quad$ [aliphatic attach]

$-\mathrm{NH}^{2}$ [aliphatic attach]

-tert Carbon [3 or more carbon attach]

$-\mathrm{N}=\mathrm{C} \quad$ [aliphatic attach]

Fused aliphatic ring unit correction

$>\mathrm{C}=\mathrm{N}-\mathrm{C}$ [cyclic-type imine, ali carbon attach]

$\mathrm{C}=\mathrm{C})-\mathrm{N}-\mathrm{)}-\mathrm{N}$ - correction

$-\mathrm{CH}=\mathrm{N}-\mathrm{C}$ [linear imine] correctionEquation Constant

$\pi$ Log Kow

$-\mathrm{CH}_{3}$ [aliphatic carbon]

$-\mathrm{CH}_{2}-$ [aliphatic carbon]

$-\mathrm{CH}^{2} \quad$ [aliphatic carbon]C

$\mathrm{C}$ [aliphatic carbon- No $\mathrm{H}$, not tert]

$=\mathrm{CH}-$ or $=\mathrm{C}<$ [olefinic carbon]

$-\mathrm{OH}$ [hydroxyl, aliphatic attach]

$-\mathrm{NH}_{2} \quad$ [aliphatic attach]

$-\mathrm{NH}^{2}$ [aliphatic attach]

-tert Carbon [3 or more carbon attach]

$-\mathrm{N}=\mathrm{C} \quad$ [aliphatic attach]

Fused aliphatic ring unit correction

$\mathrm{C}=\mathrm{C}(-\mathrm{N}-)-\mathrm{N}$ correction

$-\mathrm{CH}=\mathrm{N}-\mathrm{C}$ [linear imine] correction

Equation Constant

LogKow
1.0946

10.3131

2.5298

0.9723

1.5344

$-1.4086$

$-1.4148$

$-2.9924$

0.8028

$-0.0030$

$-2.0526$

$-1.5500$

0.6000

$-1.2000$

0.2290

2.2146

7.4546

1.6419

14.2419

2.8912

1.9446

2.3016

$-1.4148$

$-5.9848$

0.8028

$-0.0050$

$-2.0526$

$-15500$

1.2000

$-1.2000$

0.2290

4.4358

13.0458

1.6419

10.8042

2.8912

0.9723

1.5344

$-1.4086$

$-1.4148$

$-2.9924$

0.8028

$-0.0030$

$-2.0526$

0.6000

$-1.2000$

0.2290

2.71

10.40 subsequently decreases the bacterial growth of Staphylococcus aureus and Streptococcus pneumoniae bacteria.

\section{CONCLUSIONS}

The diazabicyclo-steroid derivative (compound 20) is a particularly interesting drug, 
because its antibacterial activity exerted against Staphylococcus aureus and Streptococcus pneumoniaeinvolves a molecular mechanism different in comparison with other drugs; this phenomenon may constitute a novel therapy for infectious diseases.

\section{REFERENCES}

1. Pinner, R.;Teutsch, A.; Simonsen, L.;J. Am. Med. Assoc.1996, 275, 189-193.

2. Crossley, K.; Peterson, P.;Clin. Infect. Dis.1996, 22, 209-214.

3. Chambers, H.;Emerg. Infect. Dis.2001, 7, 178-182.

4. Bogaert, D.; De-Groot, R.;Hermans, P.;Lancet Infect. Dis. 2004, 4, 144-154.

5. Yoo, B.;Triller, D.; Yong, C.;Lodise, T.;Ann. Pharmacother. 2004, 38, 1226-1235.

6. Killgore, M.; March, K.;Guglielmo, B.;Risk.;Ann. Pharmacother. 2004, 38, 1148-1152.

7. Hackbarth,C.; Chambers, H.;Antimicrob. Agents Chemother. 1989, 33, 991-994.

8. Maguire, G.; Arthur, A.; Boustead, P.; Dwyer, B.; Currie, B.;J. Hosp. Infect. 1998, 38, 273-281.

9. Parthiban, P.;Rathika, P.;Ramkumar, V.; Mo, S.; Tae, Y.; Bioorg. Med. Chem. Lett. 2010, 20, 1642-1647.

10. Oh, C.; Dong, H.; Cho, H.; Park, S.; Hong, J.; Baek, D.; Cho, J.;Arch. Pharm. Med. Chem. 2002,5, 200-206.

11. Balaji, G.; Rajesh, K.; Janardhan, R.; Vijayakumar, V.; Res. Chem. Intern. 2015, 41, 6497-6509.

12. Sedavkina, V.; Morozova, N.; Rechinskaya, A.; Kulikova, L.; Pharm. Chem J. 1974, 8, 21-24.

13. Burakova, E.;Saranina, I.; Tikunova, N.;Silnikov, N.;Russian Chem. Bull. 2015, 64, 1400-1405.

14. Arutyunyan, R.;Paronikyan, G.;Saakyan, A.; Arutyunyan, K.; Pharm. Chem. J. 2008, 42, 18-22.

15. Ponnuswamy, S.;Pushpalatha,S.;Akila, A.;Raghuvarman,B.;Aravindhan,S.; J. Mol. Struct. 2016, 1125, 453-463.

16. Aldridge, K.; Ashcraft, D.;Antimicrob. Agents Chemother. 1997, 41, 709-711.

17. Blackman, S.;Balti, R.; J. Org. Chem. 1961, 26, 2750-2755.

18. Brueggemann, A.; BKugler, A.; Doern, G.; Antimicrob. Agents Chemother. 1997,41, 1594-1597.
19. Ikee, Y.; Hashimoto, K.; Nakashima, M.; Hayashi, K.; Sano, M.;'Bioorg. Med. Chem. Lett. 2007, 17, 942-945.

20. Figueroa-Valverde. L.; Díaz-Cedillo, F.; García-Cervera, E.; Pool-Gómez, E.; LópezRamos, M.; Rosas-Nexticapa, M.;HauHeredia, L.; Sarabia-Alcocer, B.;Steroids. 2015, 93, 8-15.

21. Figueroa-Valverde, L.; García-Cervera, E.; Díaz-Cedillo, F.;Hau-Heredia, L.; RosasNexticapa, M.; Pool-Gómez, E.; LópezRamos, M.; Camacho-Luis, A.;Asian J. Chem.2016, 28, 2357-2364.

22. Halgren, T.;J. Comput. Chem. 1999,20, 720-729.

23. Nahta, R.; Yu, D.; Hung, M.; Hortobagyi, G.; Esteva, F.; Nature Clin. Pract.Oncol. 2006, 3, 269-280

24. Bièche, I.; Murcia, G.;Lidereau, R.;Clinical Cancer Research. 1996, 2, 1163-1167

25. Morris, M.; Goodsell, M.; Hallyday, D.; Huey, R.;J. Comput. Chem. 1999,19, 1639-1662.

26. Solis, F. Mathem. Meth.Oper. Res.1981, 1, 19-30.

27. Hocht, C.; Opezzo, J.; Gorzalczany, S.; Bramuglia, G.; Tiara, C.; Rev. Arg. Cardiol.1999, 67, 769-773.

28. Figueroa, L.; Diaz, F.; Ceballos G.; Lopez, M.; Maldonado, G.; Camacho, A.; J. Argentine Chem. Soc. 2008, 96, 87-100

29. Chao, K.; Rayabarapu, D.; Wang, C.; Cheng, C.;J. Org. Chem.2001, 66,8804-8810.

30. Zhao, L.; Zhang, L.; Fang, D.; Organometallics. 2016,35, 3577-3586.

31. Nishimura, A.;Ohashi, M.;Ogoshi, M.;J. Am. Chem. Soc.2012, 134, 15692-15695.

32. Goodreid, J.;Villeneuve, K.; Carlson, E.;Tam, W.;J. Org. Chem. 2014, 9, 10002-10012.

33. Uppiah, D.;Bhowon, D.;Jhaumeer, M.;EJ.Chem.2009, I, S195-200.

34. Figueroa-Valverde,L.; Díaz-Cedillo, F.; García-Cervera, E.; Rosas-Nexticapan, M.; Ramos-López, M.; Asian J. Chem. 2013, 5, 6724-6726.

35. Hania, M.;E-J. Chem. 2009, 6, 629-632. 
36. Josephsohn, N.; Carswell, E.; Snapper, M.; Hoveyda, A.;Org.Lett. 2005,7, 2711-2713.

37. Zhang, H.;Bonaga, L.; Ye, H.;Derian, C.;Damiano, B.;Maryanoff, B.;Bioorg. Med. Chem. Lett. 2007, 17, 2863-2868.

38. Reza, A.; Nasreesfahani, Z.; Ruoho, A.; Org. Prep.Proced. Int. 2008, 40, 385-391.

39. Fisher, G.; Lee, L.; Klettke, F.;Int. J. Rapid. Comm. Syn. Org. Chem. 1994, 24, 1541-1546.

40. Vedejs, E.;Fuchs P. Improved aldehyde synthesis from 1,3-dithianes. J. Org. Chem.1971, 36, 366-367.

41. Billard, F.;Robiette, R.;Pospíšil, J.;J. Org. Chem.2012, 77, 6358-6364.

42. Holum, J.;J.Org. Chem.1961, 26, 4814-4816.

43. Tokunaga, M.; Suzuki, T.; Koga, N.; Fukushima, T.; Horiuchi, A.;Wakatsuki, Y.; J. Am. Chem. Soc.2001, 123, 11917-11924.

44. Zhang, W.; Robins, M.; Tetrahedron Lett. 1992;33:1177-80.

45. Scheidt, K.; Chen, H.; Follows, B.; Chemler, S.; Coffey, D.; Roush, W.; J. Org. Chem.1998, 63, 6436-6437.

45. Newton, R.; Reynolds, D.; Finch, M.; Kelly, D.; Roberts, S. Tetrahedron Lett. 1979, 20, 3981-3982.

47. Siener, T.;Cambareri, A.; Kuhl, U.; Englberger, W.;Haurand, M.; Kogel, B.;Holzgrabe, U.;J. Med. Chem. 2000, 43, 3746-3751.

48. Weigl, M.; Wünsch, B.;Org. Lett. 2000, 2, 1177-1179.

49. Manetti, D.; Ghelardini, C.; Bartolini, A.; Bellucci, C.; Dei, S.; Galeotti, N.; Gualtieri, F. Romanelli, M.;Scapecchi, S.;Teodori, E.;J. Med. Chem. 2000, 43, 1969-1974.

50. Khrizman, A.; Slack, R.; Remsing, R.; Little,
S.;Yardley, V.;Moyna, G.;Arch. Pharm. Chem. Life.Sci. 2007, 340, 569- 576.

51. Barlocco, D.; Cignarella, G.; Tondi, D.; Vianello, P.;J. Med. Chem.1998, 41, 674-681.

52. Kinney, W.;Abou-Gharbia, M.;Garrison, D.;Schmid, J.; Kowal, D.;Bramlett, D.;Miller, T.;Tasse, R.;J. Med. Chem. 1998, 41, 236-246.

53. Lin, J.; Connelly, M.; Amolo, C.; Otani, S.; Yaver, D.;Antimicrob. Agents Chemother. 2005, 49, 1915-1926.

54. Tateda, K.; Ishii, Y.; Matsumoto, T.; Furuya, N.; Antimicrob. Agents Chemother.1996, 40, 2271-2275.

55. Pan, X.;Ambler, J.;Mehtar, S.;Fisher, L.;Antimicrob. Agents Chemother. 1996, 40, 2321-2316.

56. Calleja, C.; Pascussi, J.; Mani, J.; Maurel, P.;Vilarem,M.; Nat. Med. 1998, 4, 92-96.

57. Liu, R.; Perez, J.; Liang, D.; Saven, J.; Anesth. Analg. 2012,114, 947-955.

58. Rosales, M.; Correa, J.; Expert. Opinion Drug.Dis. 2015, 10,213-219.

59. Askew, E.; Gampe, R.; Stanley, T.; Faggart, J.; Wilson, E.; J. Biol. Chem. 2007,282,2580125816.

60. Leo, A.;Jow, J.;Silipo, C.;J. Med. Chem. 1975, 18, 865-868.

61. Leo, A.; Perspect. Drug Discov. Design. 2000,18,19-38.

62. Hansch, C.; Leo, A.; Taft, R.;.Chem. Rev. 1991, 91, 165-195.

63. Figueroa-Valverde, L.; Díaz-Cedillo, F.; Camacho- Luis, A.; López-Ramos, M.; Garcia-Cervera, E.; Monatsh. Chem. 2010, 141, 373-380.

64. Mannhold, R.;Waterbeemd, H.;J. Comput. Aided Mol. Design.2001,15, 337-354. 